\title{
Adapt, Explain, Engage-A Study on How Social Robots Can Scaffold Second-language Learning of Children
}

\author{
THORSTEN SCHODDE, LAURA HOFFMANN, SONJA STANGE, and STEFAN KOPP, \\ Cognitive Interaction Technology (CITEC), Faculty of Technology, Bielefeld University, Germany
}

\begin{abstract}
Social robots are increasingly applied to support children's learning, but how a robot can foster (or may hinder) learning is still not fully clear. One technique used by teachers is scaffolding, temporarily assisting learners to achieve new skills or levels of understanding they would not reach on their own. We ask if and how a social robot can be utilized to scaffold second-language learning of children at kindergarten age (4-7 years). Specifically, we explore an adapt-and-explain scaffolding strategy in which a robot acts as a peer-like tutor who dynamically adapts its behavior or the learning tasks to the cognitive and affective state of the child, and provides verbal explanations of these adaptations. An evaluation study with 40 children shows that children benefit from the learning adaptation and that the explanations have a positive effect especially for slower learners. Further, in $76 \%$ of all cases the robot managed to "re-engage" children who started to disengage from the learning interaction, helping them to achieve an overall higher learning gain. These findings demonstrate that a social robot equipped with suitable scaffolding mechanisms can increase engagement and learning, especially when being adaptive to the individual behavior and states of a child learner.
\end{abstract}

CCS Concepts: • Human-centered computing $\rightarrow$ Empirical studies in HCI; • Applied computing $\rightarrow$ Interactive learning environments; • Computing methodologies $\rightarrow$ Cognitive robotics; Reasoning about belief and knowledge;

Additional Key Words and Phrases: Adaptive robot tutoring, engagement, scaffolding, open learner model, transparency

\section{ACM Reference format:}

Thorsten Schodde, Laura Hoffmann, Sonja Stange, and Stefan Kopp. 2019. Adapt, Explain, Engage-A Study on How Social Robots Can Scaffold Second-language Learning of Children. ACM Trans. Hum.-Robot Interact. 9, 1, Article 6 (December 2019), 27 pages.

https://doi.org/10.1145/3366422

\section{INTRODUCTION}

The question of how educational settings with children can benefit from social robots has been receiving growing attention in recent years $[6,7]$. This is due to three major developments: first,

Thorsten Schodde and Laura Hoffmann contributed equally to this research.

This work was supported by the German Research Foundation (DFG) in the Cluster of Excellence 277 'Cognitive Interaction Technology' (CITEC) at Bielefeld University, and by the EU Horizon 2020 Program in the L2TOR (www.12tor.eu) project, Grant No. 688014, as well as the BabyRobot (www.babyrobot.eu) project, Grant No. 687831.

Authors' address: T. Schodde, L. Hoffmann, S. Stange, and S. Kopp, Cognitive Interaction Technology (CITEC), Faculty of Technology, Bielefeld University, Inspiration 1, Bielefeld, 33619; emails: \{tschodde, lahoffmann, sstange, skopp\}@techfak.uni-bielefeld.de.

Permission to make digital or hard copies of all or part of this work for personal or classroom use is granted without fee provided that copies are not made or distributed for profit or commercial advantage and that copies bear this notice and the full citation on the first page. Copyrights for components of this work owned by others than the author(s) must be honored. Abstracting with credit is permitted. To copy otherwise, or republish, to post on servers or to redistribute to lists, requires prior specific permission and/or a fee. Request permissions from permissions@acm.org.

(c) 2019 Copyright held by the owner/author(s). Publication rights licensed to ACM.

2573-9522/2019/12-ART6

https://doi.org/10.1145/3366422 
social robots have become cheaper and more robust so that practical applications are now feasible. Second, technology has matured up to a point where richer interactions using natural language or nonverbal behavior are increasingly possible. Finally, research has demonstrated that children can benefit from the presence of social robots in educational settings such as storytelling or language learning $[25,45,64,69]$. This has led to several systems already being applied in daily life, e.g., in nurseries where toy-like robots teach the alphabet to children. ${ }^{1,2}$

One rationale for employing robots, e.g., as artificial tutors, is to create learning environments that are qualitatively different from how classical educational technologies are used. It has been demonstrated that the presence of a social robot can evoke higher engagement and learning, as compared to classical on-screen media such as tablet or PC apps [33, 35, 47, 56]. This is partly due to children's tendency to be affected by social robots [82], to accept them as peers [78], or even to connect with them on a friend-like basis [40]. However, findings have also indicated that such a robot may have little or no positive effect [81], or that it can even hamper learning when producing distracting or unsettling behavior [42]. Currently, there is a lack of systematic knowledge on how a robot should (not) be incorporated in an educational setting to foster children's learning.

In this article, we ask if and how a robot could be used to scaffold the learning of children aged 4-7 years. Scaffolding refers to any strategy or temporary assistance provided by a teacher to guide and support the learner in moving toward new skills, concepts, or levels of understanding [9, 27]. More specifically, scaffolding means to build a "scaffold" for the learner by means of supportive actions, such as giving examples, demonstrating or "modeling" task solutions, highlighting the important aspects of a task, or breaking down a task into simpler sub-tasks for the learner [86]. Gibbson even argues, that "it is only when teacher support-or scaffolding-is needed that learning will take place, since the learner is then likely to be working within his or her zone of proximal development" [27, p. 16f]. If scaffolding was successful and the learner has internalized the new skill, then the scaffold can be withdrawn or adapted to lead toward skills that should be acquired next.

Scaffolding is not limited to the mere support of knowledge gain, or so-called cognitive learning $[8,48]$, e.g., by communicating needed information in suitable formats. It is also related to the learner's affective state or engagement, or affective learning [30, 49], e.g., by encouraging learners to continue or by mitigating their frustration or loss of interest [70,87]. Finally, scaffolding can also address perceived learning, e.g., by supporting the learner's self-perception of changes of skills and knowledge during learning [cf. 1, 18, p. 243ff]. These learning dimensions closely interact with each other (as discussed in the next section and summarized in Figure 2). Successful scaffolding will thus often involve addressing each of them, albeit to possibly different degrees.

We aim to study if and how social robots are capable of scaffolding learning with respect to all three of the aforementioned dimensions. We investigate this question in the domain of robotsupported second-language learning of kindergarten children ${ }^{3}$ (see Figure 1 for the learning scenario). Second-language acquisition is a complex task that involves learning vocabulary as well as prosodic patterns, syntactic structures, meanings, or situation-dependent language use [65]. It has been argued previously that the presence of a social robot that is capable of creating an interactive and motivating experience can facilitate language learning [46]. Previous studies addressed aspects such as influencing cognitive and affective learning by means of iconic gestures of the robot $[19,20]$, socially supportive behaviors [44], or an adaptive curriculum [20, 28, 71]. However, it is still largely an open question how a robot's specific actions should be designed to successfully

\footnotetext{
${ }^{1}$ https://govinsider.asia/smart-gov/exclusive-singapore-puts-robots-in-pre-schools/.

${ }^{2}$ https://www.ft.com/content/f3cbfada-668e-11e7-8526-7b38dcaef614.

${ }^{3}$ Carried out within the L2TOR project; http://www.l2tor.eu.
} 


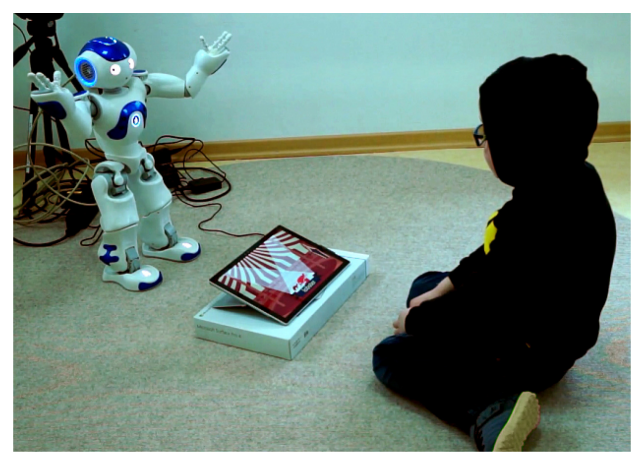

Fig. 1. Example of robot-supported language learning in kindergarten. A child learns vocabulary with a tablet app. The robot supports language learning by means of demonstrating an iconic gesture for the target words, here: lobster.

scaffold learning, especially with regard to young children's language learning, let alone how the required abilities can be technically implemented.

Our starting point is that language learning, just as any learning in general, is highly individualized and depends on, e.g., the child's changing knowledge state, social input, prior skills, uptake abilities, attention state, or engagement [4,34, 51]. A crucial component of successful scaffolding thus is to adapt both the learning task and the support to the individual child with her specific needs and abilities in the given situation. Previous research from other learning domains has shown that participants who received personalized lessons from a robot (based on heuristic assessment of the learner's skills) performed better than others who obtained non-personalized robot tutoring [56]. In previous work, we have shown that this also holds for word learning, when accompanied by a social robot that uses verbal and gestural behavior for commenting or giving feedback $[20,71]$. We also found that adapting the difficulty and order of the learning tasks improved the children's engagement and affective learning, however not their learning gain. Further, during previous evaluations with adult learners, we noticed that users at times were confused or surprised by the system's ability to estimate their knowledge and to adapt the learning task accordingly [71]. The system's scaffolding could thus have been counter-acted by the fact that learners might not have realized or understood that the learning situation has been adapted and why, which for example may have hampered perceived learning.

In this article, we present and evaluate an approach to utilize a social robot for adaptive tutoring, such that the robot provides verbal explanation to ensure transparency and understanding during the interaction to increase children's motivation and learning gain. This adapt-and-explain approach to scaffolding is based on the robot's ability to trace the knowledge state of the child and to decide about the further course of the interaction accordingly [71]. We hypothesize that perceived and cognitive learning of the children should be fostered when the robot tutor explains its decisions with its underlying beliefs about the child's knowledge state (see Figure 2), which should help the children to focus on the learning content instead of dealing with cognitive load due to uncertainty about the robot's behavior or the interaction. Additionally, we address the dimension of affective learning by investigating the robot's potential to engage children by means of simulated social cues. We hypothesize that explanations by the robot should also increase children's engagement (see Figure 2). Specifically, we look at whether dedicated re-engagement actions of the robot can help to restore engagement once it has dropped and potentially started to impede learning $[15,52]$. 


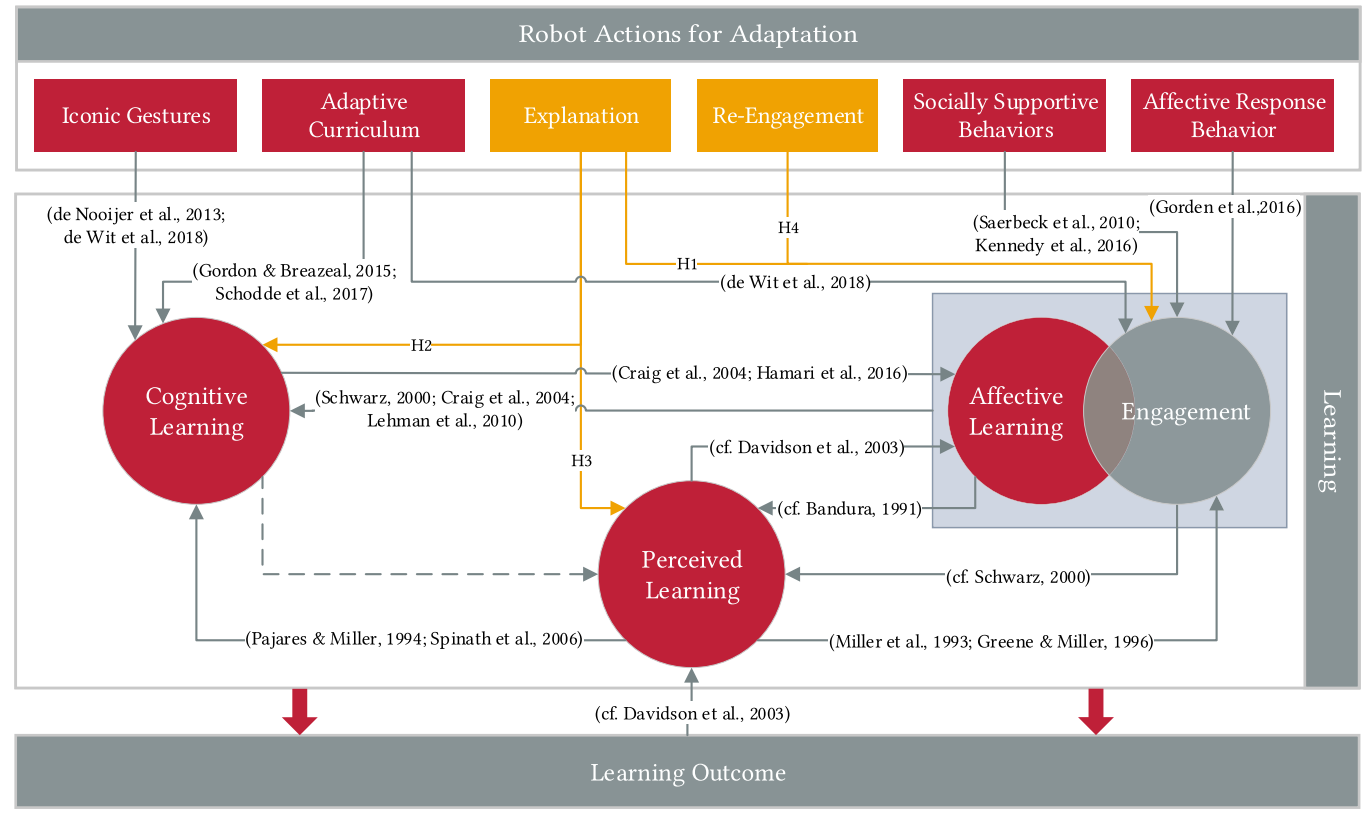

Fig. 2. Different dimensions of robot-supported learning and their interrelations. Gray arrows indicate reported correlations between dimensions of learning and engagement as well as reported effects of a robot's actions on them. The hypotheses and contributions of the study presented here are added as yellow boxes and arrows.

\section{BACKGROUND AND THEORETICAL CONSIDERATIONS}

\subsection{Dimensions of Robot-supported Learning}

Following general findings on learning, we assume that learning in robot-child tutoring involves different dimensions [43]: First, cognitive learning typically refers to the knowledge and skills to be learned [8,48]; second, affective learning represents aspects such as attitudes, values and motivation toward the object or content to be learned [30,49]; third, perceived learning relates to how much learners believe they have learned and how confident they are about their acquired knowledge [cf. 1, 18, p. 243ff]. Furthermore, we note that engagement in the overall interaction and the task is an important prerequisite for learning in robot-child tutoring $[4,51]$.

Several studies suggest that the different dimensions of learning and engagement are interrelated with each other; see Figure 2 for an overview. Consequently, most actions of a (human or robot) tutor will affect several of them: motivating the learner will lead to higher affective learning, which can increase cognitive learning. Providing good explanations during teaching can foster cognitive learning, which can lead to a more positive attitude toward the subject (affective learning) and additionally enhance the motivation to interact (engagement). This, in turn, can result in a higher learning gain and a feeling of performing well (perceived learning). Conversely, a negative attitude toward the subject of learning (affective learning) can lower engagement and thus hamper cognitive learning and then perceived learning.

As the goal of robot-supported learning is of course to maximize learning outcome, we need to optimize a robot's tutoring and scaffolding with respect to engagement as well as all dimensions of learning in the best way possible. As shown in Figure 2, different studies have demonstrated that, e.g., the use of robot gestures $[19,20]$, adaptation of the curriculum $[20,28,71]$, socially supportive behaviors [44] or affective feedback behavior [29] can facilitate learning in different ways. Here, 
we complement this picture by investigating the effects of two additional scaffolding behaviors: actions to re-engage children that become dis-engaged during the interaction, and verbal explanations of why pedagogical adaptations or actions were taken by the system. Before discussing hypotheses on the effects of these behaviors, we briefly explain how the required system capabilities for (1) adjusting the characteristics of the learning experience, (2) detecting and coping with (dis-)engagement, and (3) verbalizing the corresponding system knowledge are enabled.

\subsection{Knowledge Tracing in Tutoring Interactions}

One prerequisite for scaffolding is the ability to keep track of the learner's skill mastery, i.e., the child's learned and not-yet-learned skills. This information is indispensable to enable a personalized tutoring interaction in which the tutor needs to structure the interaction, choose the skills to be practiced, adjust the difficulty of the learning tasks appropriately, or adapt own verbal and nonverbal behavior [69]. Having access to the knowledge state of the learner allows for constructing tutoring interactions that work in the "Zone of Proximal Development" [84], which fosters flow and motivation (affective learning) and, in turn, has been demonstrated to improve cognitive learning $[15,31]$.

Previous approaches tried to trace a child's knowledge state with techniques such as Bayesian Knowledge Tracing to probabilistically model the mastery of single skills [14], Dynamic Bayesian Networks to account for skill interdependencies [41], or simple exponential functions to approximate skill knowledge [28]. Others in the field of Intelligent Tutoring Systems (ITSs) have employed approaches like Partial Observable Markov Decision Processes (POMDP) [67], Inverse Reinforcement Learning [12], or heuristic rules to model the choice of the next skill and action, while incorporating prior knowledge gathered from experts [13]. We have proposed an integrated approach that adopts Bayesian Knowledge Tracing for tracing individual skill mastery, and extends it to a model of prediction-based decision making [71]. This so-called Adaptive Bayesian Knowledge Tracing (A-BKT) allows an ITS to choose the next learning task by estimating the expected knowledge gain. The system also tries to reduce its uncertainty about the child's knowledge state, while addressing skills the child is assumed to need practice on. Evaluation studies showed that, even in a quite simple and repetitive task, adults had a significantly better learning outcome with the adaptive system [71]. For children, however, adaptive tutoring only had a positive main effect on engagement, which, however, did not mediate learning gain, probably because of too short interaction times and a lack of adaptation recognition [cf. 20]. Further, working in the "Zone of Proximal Development" means to challenge the learner with tasks they cannot complete on their own and the scaffolding might not have been sufficient [84]. Hence, one rationale for adding explanations is to provide an additional scaffold by making the underlying adaption decisions of the A-BKT model more transparent and noticeable to the child learners.

\subsection{Engagement Tracking in Tutoring Interactions}

Besides keeping track of the learner's knowledge state, managing the learner's engagement is crucial to provide an efficient and motivating tutoring experience. This is what experienced kindergarten teachers actually often concentrate on [72]. Empirical studies revealed that affective states like curiosity, interest, flow, joy, boredom, frustration and surprise can influence learner's problem-solving abilities, task engagement, and learning motivation [76]. Further, such states are found to influence cognitive processes like long-term memorizing, attention, understanding, remembering, reasoning, decision making and the application of knowledge in task solving [15, 52]. Numerous approaches have been developed to infer such states by means of analyzing, e.g., facial expressions [60], voice [21], body pressure on a seat [22], or even ECG, EEG, EMG [79, 85] and brain imaging [36]. Also multimodal approaches have been proposed, which incorporate, e.g., voice, 
interaction logs, body- and head-posture [53], or speech and text [2]. These techniques, however, are limited in their applicability to real-world interactions with children, because they are often trained on overly expressive or enacted data, require obtrusive sensor technology (e.g., wires and patches on the body), or assume a specific interaction design that, e.g., elicits speech input from the user.

In our context, engagement relates to the learner's affective and cognitive states during learning with the social robot, namely: concentration, involvement, as well as attention and interest toward both the learning material and the robot's behavior. Dis-engagement hence is defined as inattentiveness, distraction, and boredom [72]. To identify the behavioral cues most relevant to assess these states, we asked experts (kindergarteners and preschool teachers) to watch video recordings of robot-child tutoring interactions in language learning, and to elaborate on the cues based on which they assess children's dis-engagement and to name strategies that would help to restore engagement [72]. These data provided the basis for the recognition of dis-engagement (employing a human Wizard), triggering re-engaging actions, and including socially supportive behaviors (such as nodding) to prevent dis-engagement in our study (see Section 3).

\subsection{Scaffolding and Transparency Through Robot's Explanation}

Following Vygotsky's classical view, learning is to be seen as a social process: higher cognitive processes develop from observing others [75], understanding their actions, and internalizing the discovered strategies [83, 84]. To facilitate this process, a teacher can provide help through cognitive modeling: thinking aloud while solving a problem paves the way for the child to retrace the steps taken and to attain better understanding of the skill to be learned. In doing so, the teacher provides a scaffold that gives a basis from which the learner can continue on their own [55] as a form of self-regulated learning (SRL) [73, 75]. Cognitive modeling can thus help learners to develop and incorporate learning strategies by themselves, which then can be applied to new scenarios in future learning tasks [74]. In a recent approach, Ramachandran et al. [68] employed a social robot to encourage children to think aloud during the learning interaction (which is used to measure SRL [88]) and demonstrated positive effects on the learning process. This strategy also enables the teacher to provide feedback regarding learning problems. However, as children in our age group just began to show basic abilities of SRL [17], we focus on guided learning with a robot tutor by studying whether and how a robot can create transparency and scaffold learning by explaining its assessment of the child's learning (including the child's knowledge state) and its corresponding tutoring actions. This requires a model for translating the system's beliefs and decision making into spoken language explanations. We rely on the A-BKT model [71] (see above) to select from a set of predefined utterances outputted by the robot using spoken language. These explanations or comments were designed to uncover the robot's beliefs and tutoring decisions, as described in the following section (see also Section 3 for more details).

Opening up such an internal knowledge base of a system to the user is commonly referred to as an open learner model [11] or system transparency [58, 59, 61]. Open learner models often take the form of a series of skill meters $[10,38,39,57]$ and have been shown to help students to better regulate their efforts [10] or to improve their problem selection [63]. Transparency about system states has further been demonstrated to enhance trust in the system as a whole by making its behavior more understandable $[58,59,61]$ and, therefore, reducing uncertainty in the user. We thus investigate if children (aged 4-7 years) can benefit from a robot that, as an additional scaffold, uses a strategy to reveal its current beliefs about the child's knowledge state and, thereby, explains why it structures the language learning session in a particular way. We expect that this explanation will make the system's adaptations more salient, thus fostering not only engagement (see above) but also perceived learning and cognitive learning. Further, we assume that this strategy leads to a 
perception of the robot as a more competent and reasonably acting companion, which could have a positive effect on the acceptance of the whole learning environment.

\section{EXPERIMENTAL STUDY}

The aim of the present study is to investigate if and how a robot can be used to scaffold language learning in children. As in previous work, we employed the A-BKT system (see Reference [71] and Section 3.2.2) to continuously adapt the tutoring interactions (difficulty, order, number, or repetitions of learning tasks) to the individual progress of each child. Here, we study the effects of two additional scaffolding strategies that make use of the social robot. First, letting the robot give verbal explanations of its beliefs about the child's knowledge state and the according adaptations to the learning task to increase transparency of the learning interaction and inform the child's perceived learning (see Section 2.4). Second, letting the robot perform re-engaging actions when a child appeared dis-engaged to maintain engagement in the learning interaction and support learner's affective learning (see Section 2.3). To evaluate these scaffolding strategies, we conducted a study including children from three different German Kindergartens. The children had to work on English learning exercises in the form of a tablet-based animal-guessing game in the presence of a social robot that acted as a knowledgeable peer controlling the tablet (see Figure 1 for the overall setting).

We used a one-factorial between-subjects design where the children either interacted with a robot that explained its decisions and beliefs about the child's knowledge before the target word was announced, or not. The children were randomly assigned to one of the conditions, balanced for gender and age. In both conditions the robot performed dedicated re-engagement behaviors, whenever a human Wizard recognized cues of dis-engagement.

Based on findings from work on open learner models [11] and transparency [58, 59, 61], we hypothesized that verbalizing the robot's belief during the tutoring interaction will make the interaction more meaningful, which will increase the children's motivation to continue interacting (H1), overall learning gain (H2), and perceived learning (H3). For the re-engagement actions, we hypothesized that children who display dis-engagement will re-focus and continue learning after a re-engaging act by the robot (H4a), especially if the initiation of such an action is additionally explained by the robot (H4b, indicated also in Figure 2).

H1: Children who interact with a robot tutor that explains its belief about the child's knowledge are more motivated to continue learning than children who interact with a robot that does not provide such explanations.

H2: Children who learn with a robot tutor that explains its belief about the child's knowledge show a stronger learning gain than children who interact with a robot that does not provide explanations.

H3: Children who learn with a robot tutor that explains its belief about the child's knowledge gather a better understanding of their perceived learning than children who interact with a robot that does not provide explanations.

H4a: Dis-engaged children will be re-engaged through dedicated actions executed by the robot.

H4b: Dis-engaged children will be more re-engaged when the robot explains its decision to start a re-engagement attempt.

\subsection{Participants}

In total, $n=49$ children ( 22 female, 27 male) between the ages of 4 and 7 years from three different kindergartens in Germany participated in the tutoring interactions. Parents were informed in advance about the purpose and procedure of the study and had to sign consent if their child was willing to participate. The children were randomly assigned to one of the two conditions, 
A

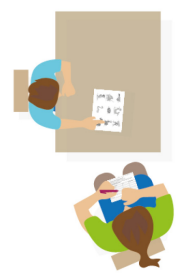

B

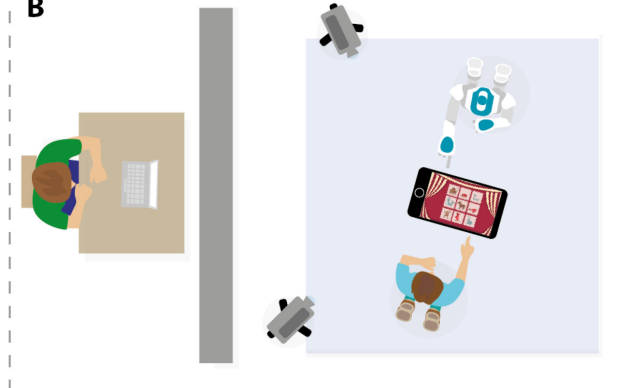

C

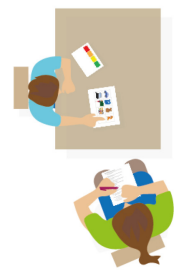

Fig. 3. The experimental setup including (A) a paper and pencil pre-test, (B) the child-robot interaction supervised by technician, and (C) a post-interaction interview in a separate room.

balancing age and gender within the conditions. Nine cases had to be removed due to incomplete datasets, resulting from system crashes $(n=2)$, early termination of the interaction due to annoyance or anxiety displayed by the children $(n=6)$, and one case because of impairments in language development that might have affected the overall communication with the robot $(n=1)$.

This leads to a remaining sample of $n=40$ children (20 female, 20 male), aged 4-7 $(M=$ $5.43 ; S D=0.54$ ), whereof 22 children participated in the experimental (with-explanation) and 18 in the control group (without-explanation).

\subsection{Materials}

The experimental setup for the robot-child tutoring interaction included a robot (Softbank Robotics' Nao Version 5), and a Microsoft Surface tablet computer. The interaction was monitored by a technician in the same room, however, out of the child's field of view. In addition, two video cameras recorded the interaction from different angles (see Figure 3) for post hoc behavior analyses. ${ }^{4}$

3.2.1 Scenario and Chosen Vocabulary. To challenge, but not overwhelm the children with the amount of input, we presented them with nine English animal names as target words. The underlying interaction was framed in a child-friendly way, being that the robot and the child are visiting a virtual circus together. A circus is commonly known and liked by children [cf. 37] and provides a reasonable frame for different animals showing up in the same place. Furthermore, we took careful decisions in the animal selection based on theoretical assumptions and lessons learned from earlier experiments. For example, we did not include the word "chicken," since we remarked earlier that it is widely known by kindergarten children. Further decisive factors were (a) dissimilarity between German- (first language: L1) and English-word (second language: L2), (b) the possibility to create a comprehensible iconic gesture linked to the animal, e.g., scratching like a monkey (see Figure 4) that is executable by the robot, and (c) choice of groups of animals that can be presented in similar colors.

We finally chose: parrot, lobster, and ladybug as red-colored; rabbit, snake, and seal in gray; bull, monkey, and horse for the brown-colored ones as target words for the tutoring interaction (see Figure 6(D)). Similar to our previous study [20], the task difficulty was varied by means of different amounts of distractors, i.e., presentation of three, six or nine different animals on the screen. Additionally, we considered the color as an influencing factor, resulting in four levels of difficulty from easy to hard: 3 animals with 3 different colors, 3 animals with the same color, 6 different animals ( 2 red, 2 gray, 2 brown), and 9 different animals. Colors were important for

\footnotetext{
${ }^{4}$ The study software can be downloaded at https://pub.uni-bielefeld.de/record/2938068.
} 

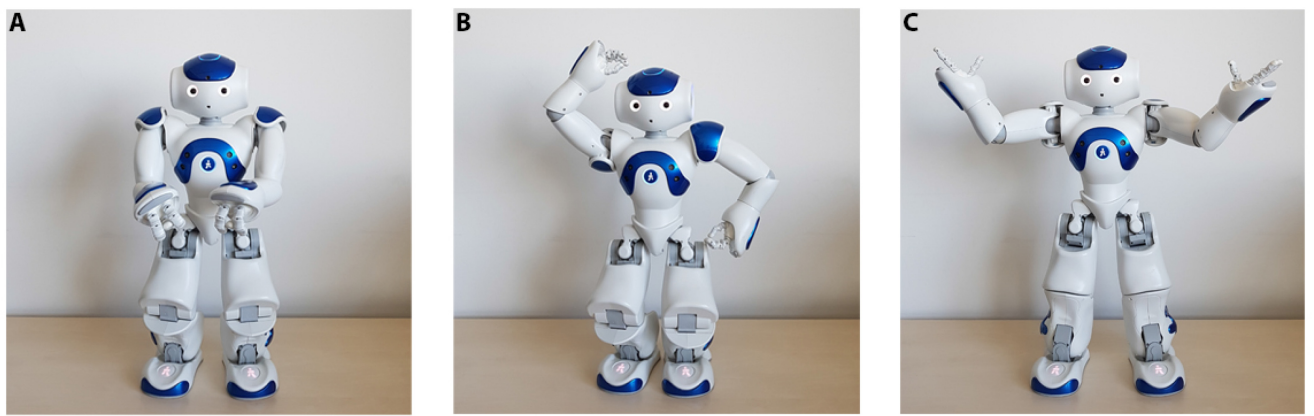

Fig. 4. Examples of the iconic gestures shown by the robot for the animals used in the experiment. (A) Imitating riding a horse by grasping imaginary reins and moving the arms up and down; (B) imitating a monkey by scratching hip and head; (C) imitating a lobster by raising the arms and opening/closing the hand like lobster-claws.

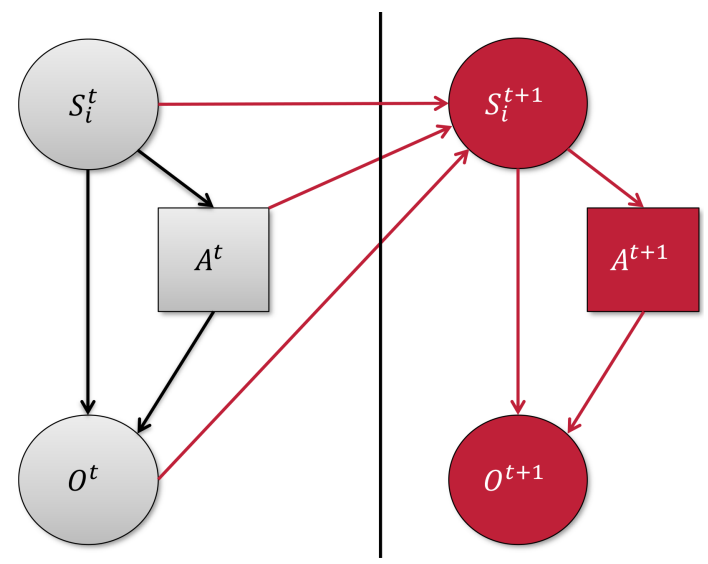

Fig. 5. The Adaptive Bayesian Knowledge Tracing (A-BKT) model. $S_{i}^{t}$ represents the system's belief about the learner's skill mastery for skill $S_{i}$ at time $t$. After a teaching action $a^{t}$ is executed, the system observes an answer $o^{t}$ and updates the skill belief correspondingly.

our choices as they are a salient feature of objects for children, and might thus result in children remembering the color of the animal instead of the target word [5]. This is why we included different animals with the same color.

3.2.2 A-BKT Model. As mentioned before, the developed A-BKT model is based on Bayesian Knowledge Tracing (BKT) [14]. Traditionally BKT is represented by a Hidden Markov Model and consists of a binary latent variable $S_{i}^{t}$ (mastered or not mastered) representing the system's belief to which extend the skill $S_{i}$ has been mastered by the learner. This belief state is updated based on the observation $O^{t}$ that corresponds to the learner's response to a learning task, e.g., correct or wrong answer to a question. The update process is further guided by the probabilities of guessing $p$ (guess), i.e., answering correctly while not having mastered the skill yet, and slipping $p$ (slip), i.e., answering wrongly although knowing the skill.

In previous work this model was extended to also include an action decision node $A^{t}$, which allows to model the ITS's decision-making process based on the current beliefs about the learner's knowledge state ([71], Figure 5). Additionally, the latent variable $S_{i}^{t}$ was modified so that it can 
attain six values for each skill, corresponding to six bins of the belief state $(0 \%, 20 \%, 40 \%, 60 \%$, $80 \%, 100 \%$ ). These changes allow to quantify the learner's uncertainty in more detail, as well as to model/simulate the impact of tutoring actions on the future observations and the skill development.

Consequently, the A-BKT model is not only able to trace the learner's knowledge state of different skills, while for each skill an own network is used, but also to take out decisions about the following course of the tutoring interaction. Both aspects are explained in more detail in the following.

Knowledge tracing: To update the system's belief about a specific skill $S_{i}$ after observing an answer $o^{t} \in O^{t}$ to a tutoring action $a^{t} \in A^{t}$, a simple Bayesian update rule is employed for each $s_{k} \in S_{i}^{t+1}$ :

$$
\begin{aligned}
p\left(s_{k}\right) & :=p\left(s_{k} \mid o^{t}, a^{t}\right) \\
& =\sum_{s_{j} \in S_{i}^{t}}\left[p\left(s_{j} \mid o^{t}, a^{t}\right) \cdot p\left(s_{k} \mid s_{j}, o^{t}, a^{t}\right)\right] \\
& =\sum_{s_{j} \in S_{i}^{t}}\left[\frac{p\left(o^{t} \mid s_{j}, a^{t}\right) \cdot p\left(a^{t} \mid s_{j}\right) \cdot p\left(s_{j}\right)}{p\left(o^{t}, a^{t}\right)} \cdot p\left(s_{k} \mid s_{j}, o^{t}, a^{t}\right)\right] .
\end{aligned}
$$

Predictive decision making: The decision-making process consists of two parts. First, the next skill to address needs to be selected and, second, an appropriate tutoring action (task difficulty) to address this skill needs to be chosen. For the former, the Kullback-Leibner divergence (KLD) is used to compare each traced skill belief $S_{i}^{t} \in \mathbb{S}$ with the desired end belief state $S_{\text {opt }}$ :

$$
\text { next_skill }=\underset{S_{i}^{t} \in \mathbb{S}}{\operatorname{argmin}}\left[\alpha\left(S_{i}^{t}\right) \cdot K L D\left(p\left(S_{i}^{t}\right), p\left(S_{\text {opt }}\right)\right)\right] .
$$

Since the KLD requires at least some probability mass in each bin, $S_{o p t}$ is defined by containing $99.999 \%$ of the probability mass in the last bin (100\%), meaning, being $99.999 \%$ sure that the learner mastered skill $S_{i}$ to $100 \%$.

This algorithm results in selecting skills more often with which the learner struggles most, since for these skills the system's belief state will be the lowest. To prevent that a specific skill is taught over and over again, $\alpha\left(S_{i}^{t}\right)$ is added, which ranges from 0.0 to 1.0 . It is decreased by 0.3 each time a specific skill $S_{i}$ is addressed and increased again by 0.15 if another skill is practiced. The resulting skill selection behavior is comparable to the spaced repetition method as implemented in the Leitner system [54, p. 64ff.].

After a skill has been selected, the system has to choose the next tutoring action to optimally address this skill. Similar to the skill selection, the action selection is also modeled as a minimization problem:

$$
\text { next_action }=\underset{a_{l} \in A^{t}}{\operatorname{argmin}}\left[\beta\left(S_{i}^{t}, a_{l}\right) \cdot K L D\left(p\left(S_{i}^{t+1} \mid a_{l}\right), p\left(S_{o p t}\right)\right)\right],
$$

where

$$
\begin{aligned}
p\left(S_{i}^{t+1} \mid a_{l}\right) & :=\sum_{o_{m} \in O^{t}} \sum_{s_{j} \in S_{i}^{t}}\left[p\left(s_{j} \mid o_{m}, a_{l}\right) \cdot p\left(s_{k} \mid s_{j}, o_{m}, a_{l}\right)\right], \forall s_{k} \in S_{i}^{t+1} \\
& =\sum_{o_{m} \in O^{t}} \sum_{s_{j} \in S_{i}^{t}}\left[\frac{p\left(o_{m} \mid s_{j}, a_{l}\right) \cdot p\left(a_{l} \mid s_{j}\right) \cdot p\left(s_{j}\right)}{p\left(o_{m}, a_{l}\right)} \cdot p\left(s_{k} \mid s_{j}, o_{m}, a_{l}\right)\right], \forall s_{k} \in S_{i}^{t+1} .
\end{aligned}
$$

As can be seen, the direct inclusion of the tutoring action into the Bayesian network allows to simulate possible outcomes for all actions $a_{l} \in A^{t}$ and, based on this, to choose the action that will 
Table 1. Overview of Dis-engagement Beliefs, Related Cues, and Repair Actions Used in the Study

\begin{tabular}{l||l|l}
\hline Dis-engagement Belief & \multicolumn{1}{|c}{ Behavioral Cues } & \multicolumn{1}{c}{ Repair Actions } \\
\hline Tired & Rub eyes, Yawn, & Joint breathing, \\
& Support head with hands & Stretching \\
\hline Heightened activity & Move position, & Stand up and squat, \\
& Undirected finger-tapping & Stand up and lift arms \\
\hline Distraction low & Gaze away (from robot \& tablet), & Wave, \\
& Grimace & Whisper \\
\hline Distraction high & Turn away, & Chicken dance \\
& Move head from left to right & \\
\hline
\end{tabular}

be most likely profitable for the learner. To simplify this process, $\beta\left(S_{i}^{t}, a_{l}\right)$ is introduced, which is based on a series of overlapping Gaussian curves distributed over the full belief space of a skill $S_{i}$, while each curve represents the field of application for a specific action $a_{l}$. This allows for $\beta\left(S_{i}^{t}, a_{l}\right)$ to modify the KLD so that it is higher if an action is selected, which is assumed to be inappropriate for the current skill belief (e.g., high task difficulty for low skill mastery). However, if an action is assumed to perfectly fit the current skill belief for $S_{i}$, then $\beta\left(S_{i}^{t}, a_{l}\right)$ is 1.0 and does not affect the KLD. Overall, this definition results in a model that allows to adapt to the learner's individual learning needs by selecting an easy task if the skill mastery is believed to be low, a hard task if it is high, and medium in-between.

3.2.3 Improvements of the Tutoring Experience. The underlying system used in the present study is based on our previous work with kindergarten children and teachers [20,71], and our experiences on how to design a beneficial learning interaction for this particular age group [7]. In particular, we devised a new feedback strategy, new robot behaviors to heighten children's engagement, and new verbal explanations of the robot's current belief about the child's knowledge state.

Feedback strategy: Based on previous observations [7,71], the robot gives explicit positive feedback, e.g., praising the child, when receiving linguistic input and when the right answer is selected. If a wrong answer is given, instead of uttering negative feedback, then the target word is repeated by the robot, followed by its translation in L1. Afterwards, only the wrongly chosen image and the target image are presented and the child is invited to choose once again.

Actions to prevent dis-engagement: In line with suggestions from experts on how to increase children's engagement [72], we implemented small rewarding behaviors of the robot, such as gazing toward the child or nodding when verbal input is received. To signal positive feedback each time the child answered correctly, the robot's eyes were lighted up, blinking in rainbow colors to resemble a smiling face (as has already been used by Fridin [25]). Other than that, iconic gestures for each animal were included to increase children's interest in the interaction (cf. Reference [20]). However, this time, gestures were not displayed each time an animal name was mentioned by the robot, but only executed once for each animal in the introduction. Moreover, to prevent children from repeating already known words over and over again, the adaptive system is now allowed to terminate the interaction early if it assumes an average probability of $75 \%$ or higher that a child learned the target words.

Actions to restore engagement: In addition to the preventive actions-executed by the robot regardless of the child's state-we designed repair actions that could be carried out spontaneously during the interaction when drops of engagement were observed. We focused on the cues derived from expert interviews to detect dis-engagement [72], summarized in Table 1. As automatic 
detection is not feasible at the moment, we included a human Wizard of $\mathrm{Oz}$ [16] who kept track of the child's behavior.

The Wizard was trained beforehand to be aware of the corresponding cues. As soon as the child displayed a behavioral cue of dis-engagement, the Wizard selected a corresponding type of disengagement category (see Table 1): tired, heightened activity, low and high distraction. For each of these categories, a set of repair actions were assigned to be executed by the robot to re-engage the child. These actions ranged from simply breathing deliberately and stretching, when the child seemed to be tired, to standing up and moving the arms, or doing squats when the child seemed to have excess energy. All repair actions were primarily executed by the robot, however, most of them invited the child to actively participate, e.g., stand up and squat. In cases where the child appeared only slightly distracted, e.g., by gazing away or grimacing, the robot would just wave or whisper to regain attention. When the child would display strong cues of distraction, such as turning completely away or moving away from the interaction, the robot would try to motivate the child to join a dance as a last resort. These actions were designed to move the children's attention toward a different aspect, but subsequently direct it back toward the vocabulary learning task to restore the engagement. By noticing, acknowledging, and reacting to a decrease in engagement, the robot should help getting back the child's attention [72].

Explanation Verbalization: The robot's explanations of the adaptive tutoring are based on the A-BKT model, which continuously keeps track of the child's proficiency level (per target vocabulary). These system beliefs are defined as probabilities of having learned the corresponding skill/word. They provide the basis for selecting the next learning task and determining its difficulty (distractor objects, arrangement on the tablet) as described in Section 2.2. The underlying proficiency belief is discretized into four levels, which are verbally explained by the robot as follows:

(1) very low proficiency (belief about skill: $\sim 0-25 \%$ ), very easy task:

e.g., "For practice, I'm selecting an animal which I believe you don't know yet. To keep it simple, only three different animals stand for selection."

(2) low proficiency (belief about skill: $\sim 25-50 \%$ ), easy task:

e.g., "I'm selecting an animal that I believe you are not completely certain about yet. Try and find it among the three animals of the same color"

(3) medium proficiency (belief about skill: $50-75 \%$ ), medium hard task:

e.g., "Next, we are repeating an animal that I am quite sure you know. To make it a little more challenging, you have to choose from six animals."

(4) high proficiency (belief about skill: 75-100\%), hard task:

e.g., "I'm now selecting an animal that I am very certain you know. To strengthen your knowledge, it is hidden between eight other animals. I am curious to see whether you find it."

These sentences occur only in the with-explanation-condition and are followed by the typical "I spy with my little eye and see something that is a [target word]," uttered in both conditions. Further, the four wizarded beliefs about the learners' (dis-)engagement are verbally made explicit by the robot:

(1) tired: "I am under the impression that you are a little tired."

(2) activity: "I think you cannot concentrate anymore."

(3) distraction low: "I have the feeling that you are a bit distracted."

(4) distraction high: "I think that you are too distracted to continue learning right now."

These phrases serve as an explanation for the respective re-engagement behavior, given only in the corresponding explanation condition, and are followed by the robot inviting the child to join in on a dedicated activity, in both conditions. 
The explanations are meant to reveal the system's perception of the children's performance and the resulting consequences for the difficulty and course of the learning interaction to the children themselves.

\subsection{Measurements}

3.3.1 Engagement. Multiple factors are considered to assess children's engagement. First, the frequency of dis-engagements: whenever a child showed signs of dis-engagement, the Wizard triggered a re-engagement behavior. It was noted how many children in total showed signs of disengagement, and how many attempts to re-engage occurred per child. Second, since the children were instructed that termination of the interaction is possible at any given time, the number of rounds they played are regarded as a further indicator of interaction engagement. Yet, since there were diverse reasons for an early termination of the game, a shorter interaction time does not necessarily account for a lack of engagement, e.g., if the system decided to end early due to a good performance of the child. Therefore, different reasons for the termination of the interaction were taken into account: (1) The child decided to quit after being asked whether she wants to continue after a re-engagement attempt, (2) the WOz triggered the fourth re-engagement behavior, which was set as a threshold, leading to a termination of the interaction, (3) the system decided that the child has learned well enough and therefore ended the interaction, (4) a maximum number of 30 rounds has been reached, and (5) the child quit by herself without being asked by the robot (after a re-engagement action).

3.3.2 Learning Gain. To measure the children's learning gain, a pre-, post-, and retention-test were conducted. The pre-test was carried out as follows: first, the child was presented with a paper sheet displaying pictures of all 9 animals in random order. The child was then asked to name the respective animals in its L1, to ensure all presented animals are known beforehand. Afterwards, the experimenter verbally presented the animal names in the second language (English), requesting the child to tap the corresponding picture with their finger. In the meantime, the experimenter noted whether the child answered correctly, but did not provide feedback about the performance to the child.

Subsequent to the interaction, post- and retention-test were used to measure the knowledge gain for the target words. While the post-test was conducted right after the teaching interaction, the retention-test took place at least 1 week later, where both used the same layout and procedure. After the child had received an explanation of the task from the robot and stated to have understood the task, the robot started to present the animal names in the second language, while all animals were displayed on the tablet screen. The child was asked by the robot to feed a grape to the animal whose name was announced. For this purpose, the child had to gather a virtual grape from a basket on the bottom right of the screen and move it to the intended animal to feed it (see Figure 6(D)). Note that during all three tests the same animal pictures were used and the children only got neutral feedback (e.g., Thanks. The next animal we feed is [target word]), so that they did not know if their answer was correct or not.

3.3.3 Perceived Learning. To asses how the children estimate their knowledge state regarding the target words, we presented children again with printed pictures of the animals and asked them to sort them into four knowledge categories according to the discretized knowledge levels in the system $(0-25,25-50,50-75,75-100 \%)$. The categories were discernible via colored squares onto which children were asked to place the pictures, along with a verbal explanation of each category by the experimenter. The colors were:

- red: "I don't have a clue what the English name of this animal is."

- orange: "I'm uncertain what the English name of this animal is." 

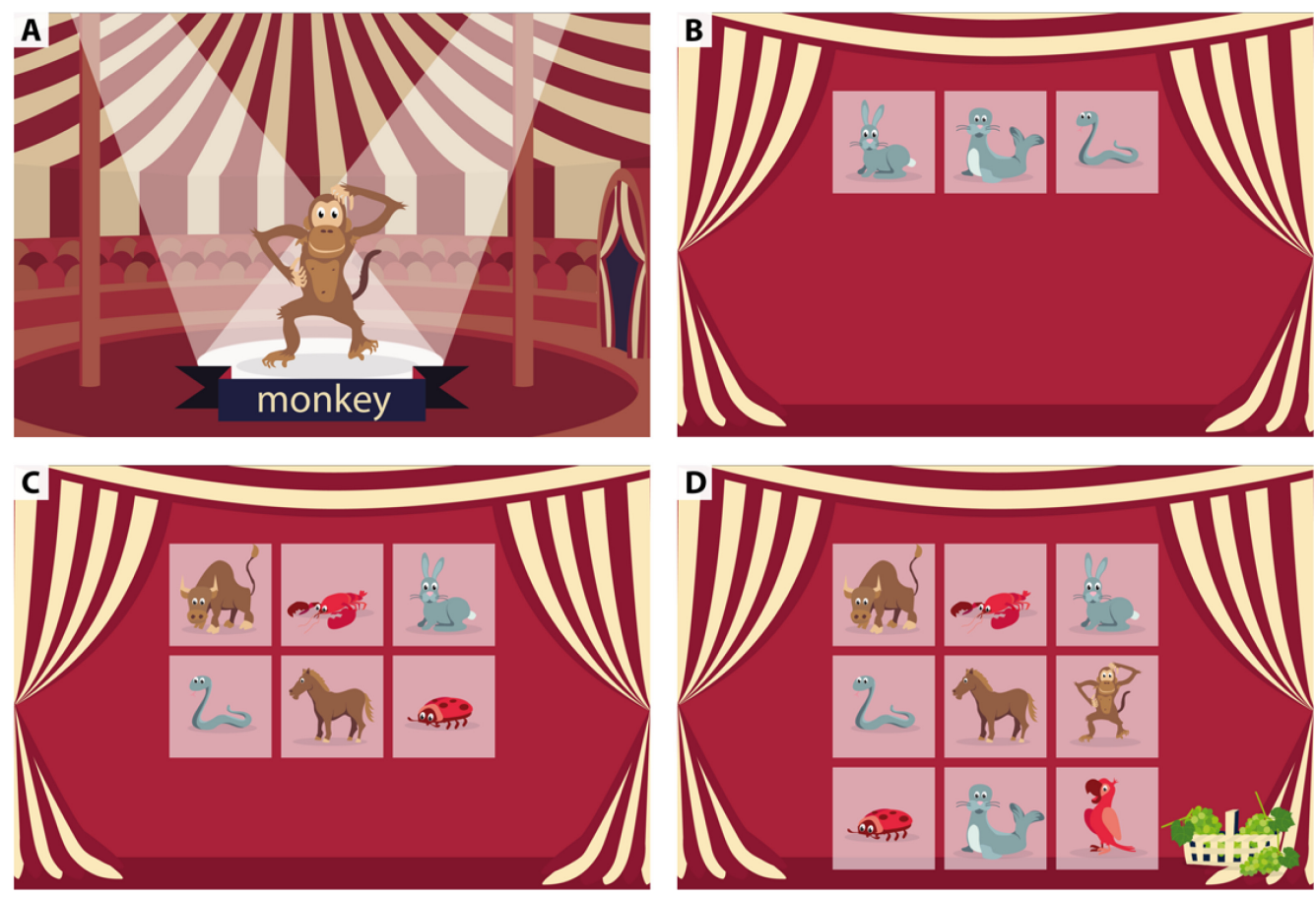

Fig. 6. Screen captures of the learning interaction displayed on the tablet including (A) the introduction of a new animal, (B) and (C) the presentation of a choice of animals of task difficulty 1 and task difficulty 3 during training, (D) the post-test including all target words.

- yellow: "I'm rather sure what the English name of this animal is."

- green: "I definitely know the English name of this animal."

After having explained the procedure, the experimenter handed over card by card, while each card displayed a printed version of the animals included during tutoring. The child was requested to put each card to the one category that best fitted their own knowledge estimation, while being advised that they could put as many cards as they wish into one category. For further comparisons of the children's estimated knowledge to their answers in the post-test (which was either coded as right $=1$, or wrong $=0$ ), we collapsed the categories into high perceived knowledge (green and yellow) and low perceived knowledge (orange and red).

3.3.4 Re-engagement Success. As a measure for re-engagement success, we considered continuation of the interaction after an attempt to re-engage a child. Since children were asked whether they want to continue learning after each re-engage attempt, we took their decision to continue as an indicator of successful re-engagement. If children quit the interaction early after several attempts of re-engagement, then we counted all attempts that resulted in continuation as successful attempts (per child), but the last attempt as unsuccessful.

\subsection{Procedure}

A few weeks before the study, information about the goal, setting and course of the study were handed out to the parents of the children in the respective age group. The parents were informed that for the children to be able to participate a consent form had to be filled out, and encouraged to contact the experimenters in case of any questions. 
One week before the actual study took place, the experimenters visited the preschools to introduce the robot and themselves to the children in a group session. This approach was inspired by the work of Vogt et al. [80] and Fridin [24] who reported positive effects of a first meeting of the robot in a safe and known environment to overcome initial anxiety before interacting with the robot one-on-one. The introductory session was designed as a conversation between the experimenters, the robot and the children. The experimenters began presenting themselves followed by a short background story of the robot, which remained initially static. The children were then encouraged to describe the robot's body parts. Here, the similarities (arms, legs, etc.) and dissimilarities (especially face and fingers) between the robot and actual humans were highlighted. The children were then motivated to collectively wake the robot by calling its name. Afterwards, the robot started greeting the children and told a few facts about itself. The robot also suggested to dance the chicken dance to loose its tension. The sound of the chicken dance was then played and robot and children danced together to the sound. Finally the robot went back to a kneeling position to rest. The children were then free to touch the robot carefully and ask further questions until the first curiosity was satisfied and the robot said farewell.

On the actual study day, each child entered the room (either with a teacher or alone) and sat down at a small table together with an experimenter. After the child had been informed that they could stop at any time they want, the pre-test was conducted (see Figure 3(A)). Subsequent to the pre-test, the child was guided to the main interaction spot and asked to sit down in front of the tablet and robot on the ground (see Figure 3(B)). The experimenter then briefly explained how to use the tablet and that the child was about to learn English vocabulary while visiting a virtual circus together with the robot.

The robot then started with light conversation, asking for the child's name, age and previous knowledge of English vocabulary (see Figure 9 for details on the robot's actions). Afterwards the circus was introduced as well as all animals living in this circus, one after the other. While the German and English animal names were presented verbally by the robot, the English name and a picture of the selected animal were presented visually on the tablet (see Figure 6(A)). This input was further enriched by iconic gestures executed by the robot as it has been shown in an earlier study that word learning is supported by accompanying gestures [20]. The child was further requested to repeat the English name of the animal to strengthen the recall.

After all animals had been introduced, the real learning game, a modified version of the children's game "I spy with my little eye..." was explained. To verify if the child understood the game principle, two test rounds were played before the 30 rounds of learning finally started. In each round the adaptive system chose the skill to teach, i.e., an English animal name, in a given task difficulty, expressed through different amounts of animals displayed on the tablet screen (see Figures $6(\mathrm{~B})$ and $6(\mathrm{C})$ ). The child then had to tap the animal on the screen that depicted the animal announced by the robot (in English).

After 30 rounds, or as soon as any other reason for the termination of the interaction occurred (see termination reasons), the post-test started (see Figure 6(D)). After all nine animals had been fed, the robot thanked the child for playing with it and said goodbye. Then the experimenter came back and gave grapes to the child and asked if they want to share them with the robot. Finally, a picture of the child and the robot was taken as a souvenir for the child, before the final interview was initiated by the experimenter, in which children were asked to estimate their perceived learning of the animals (see Figure $3(\mathrm{C})$ ).

At least one week later, the children met the robot again for the retention-test. As in the posttest, they were requested again to feed all 9 animals and afterwards the robot thanked the children for playing with it and said goodbye. 


\section{RESULTS}

In total, data of $n=40$ children at the age of four to seven years were analyzed for the impact of the scaffolding strategies on understanding, motivation, learning gain, and willingness to continue after dis-engagement. ${ }^{5}$

\subsection{Children's Engagement}

As indicators of children's engagement, we considered (1) the frequency of behavioral cues of disengagement (e.g., yawning or gazing away; see Table 1) during the interaction, (2) the number of rounds children played, since they were free to terminate the interaction at any given time, and (3) the reasons for termination.

Based on our earlier experience [20,72], we expected children to become easily dis-engaged after a couple of rounds due to their short attention spans [50]. However, only twelve children ( $n=7$ in explanation condition, $n=5$ in control group) displayed one or more behavioral cues of dis-engagement at all. For instance, children played with their clothes or stood up and sat down in a different posture again while not paying attention to the tablet or robot. A comparison of the occurrence of dis-engagement between the conditions revealed that the difference was not significant.

This was somehow unexpected as the tutoring interaction lasted on average more than 18 minutes in the control condition $(M=18.16 ; S D=2.18)$ and 23 minutes in the explanation condition $(M=23.76$ minutes, $S D=3.97)$, which is even longer than in previous studies in which children's engagement dropped significantly [20]. To determine whether the mean differences were of statistical significance, we compared the means with an independent sample t-test, after having ensured normal distribution (with Shapiro Wilk's test) and homogeneity of variances (with Levene's test) between the conditions, which both turned out to be non-significant. The analysis revealed that the interactions were in fact significantly longer in the explanation condition $\left.\left[t(38)=5.36 ; p<.001 ; d_{\text {Cohen }}=-1.70\right)\right]$. However, we did not observe more dis-engagement or more early termination in this condition. The average number of rounds children played with the robot with explanation were $M=26.55, S D=5.37$, without explanation $M=27.67, S D=3.58$. In principle, this could hint at a stronger engagement in the explanation condition, in which the overall duration of interaction was much longer. This, however, is not true when looking at the numbers of terminations.

Every interaction was terminated, because (1) children decided to quit either by themselves or after being asked by the robot whether to continue after a re-engagement attempt, (2) the system decided to stop after four recognized cues of dis-engagement, (3) the system decided to terminate early due to a high belief that the child's knowledge was high enough (above 75\%), or (4) the child reached a maximum of 30 rounds. As visible from Table 2, the majority of children played all 30 rounds or finished early due to a high skill mastery belief, whereas only a few dismissed after attempts to re-engage them. A comparison of the frequency of termination reasons by means of Pearson's chi-squared test between the conditions yielded no significant differences: children continued or dismissed similarly often, regardless of the robot's explanation behavior.

In sum, H1 was not confirmed as children did not appear more engaged when the robot explained its beliefs or decision making, as visible from children's signs of dis-engagement, continuation of playing, and reasons to terminate.

${ }^{5}$ The recorded dataset can be downloaded at https://pub.uni-bielefeld.de/record/2938068. 
Table 2. Numbers of Children Grouped According to Experimental Conditions and Reasons for Termination of the Interaction

\begin{tabular}{l|c|c|c}
\hline Termination reasons & $\begin{array}{c}\text { With Explanation } \\
(\mathbf{n}=\mathbf{2 2})\end{array}$ & $\begin{array}{c}\text { Without Explanation } \\
(\mathbf{n = 1 8})\end{array}$ & $\begin{array}{c}\text { Children } \\
\text { in total }\end{array}$ \\
\hline Played 30 rounds & $11(50 \%)$ & $11(61.11 \%)$ & 22 \\
\hline $\begin{array}{l}\text { Finished early due to high } \\
\text { knowledge state (system's decision) }\end{array}$ & $7(31.82 \%)$ & $5(27.78 \%)$ & 12 \\
\hline $\begin{array}{l}\text { Finished early after re-engagement } \\
\text { attempt (child's decision) }\end{array}$ & $2(9.1 \%)$ & $2(11.1 \%)$ & 4 \\
\hline $\begin{array}{l}\text { Finished early after 4 attempts to } \\
\text { re-engage (system's decision) }\end{array}$ & $2(9.1 \%)$ & - & 2 \\
\hline
\end{tabular}

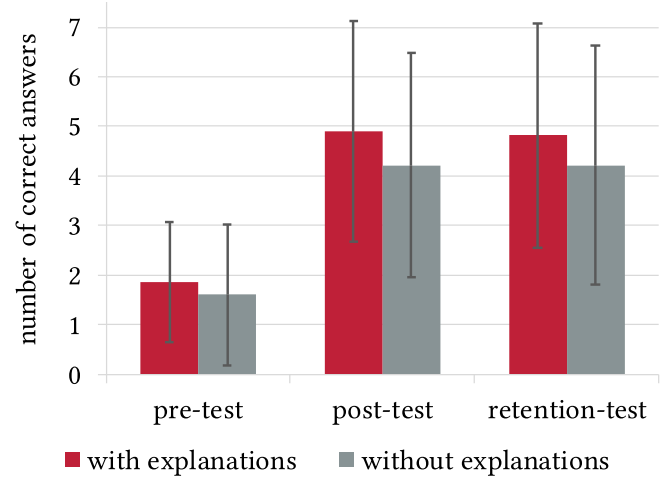

(a)

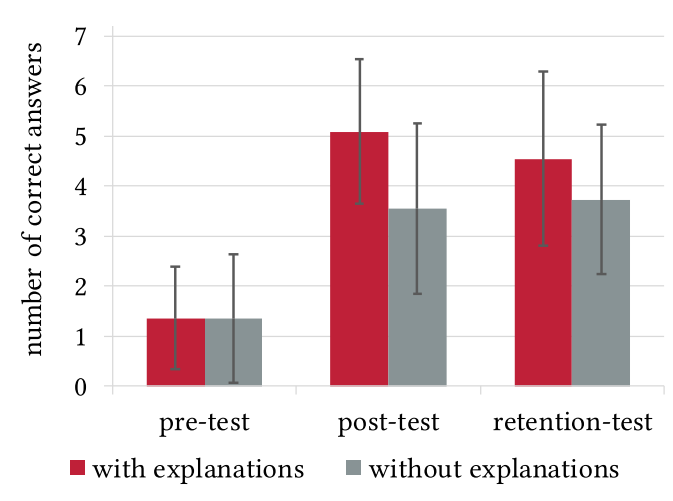

(b)

Fig. 7. Average numbers of correct words in pre-, post-, and retention test: (a) all children $(n=40)$ but with different durations of interaction; (b) only children who played 30 rounds ( $n=22 ; 11$ in each condition).

\subsection{Learning Gain and Persistence}

To measure learning gain and its persistence, we administered tests of the to-be-learned words before, immediately after, and 1 to 4 weeks after the tutoring interaction (due to holidays in kindergartens). For the latter retention tests, an independent samples t-test showed no significant difference between the average delays between conditions (without-explanation: $M=11.39$ days; $S D=$ 6.61 days; with-explanation: $M=12.95$ days; $S D=7.05$ days). Overall, the average numbers of correct answers in the with-explanation condition was higher than without explanations, at each of the different test times (pre/post/retention; see Figure 7(a)).

Because of large variances in the absolute values already observable in the initial pre-test, we calculated the learning gain as the difference between the pre- and post-test, and the persistence of the learning gain as the difference between post- and retention-test. Results show a higher learning gain in the condition with explanations $(M=3.05 ; S D=2.19)$ as compared to the condition without explanations $(M=2.61, S D=2.00)$. In contrast, the learning gain persistence appeared slightly higher in the condition without explanations $\left(M_{\text {without }}=0.00, S D_{\text {without }}=\right.$ $\left.1.82 ; M_{\text {with }}=-0.09, S D_{\text {with }}=1.95\right)$. To compare the mean learning gain and its persistence between the experimental conditions, we analyzed the variables with respect to their distribution and homogeneity of variance. As Shapiro-Wilk's and Levene's tests yielded no significant results, we used independent sample t-tests. However, all differences were not significant. 


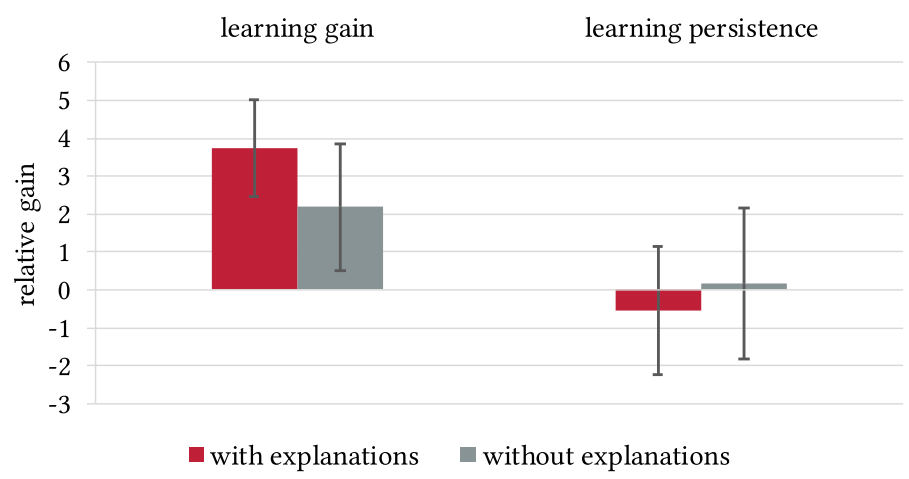

Fig. 8. Average learning gain and persistence for children who played all 30 rounds ( $n=22 ; 11$ in each condition).

Because of the high standard deviations, we investigated further causes that might have affected children's learning. Since the adaptive design of the interaction resulted in a variety of termination points (from after 9 to after 30 rounds), for the sake of comparability, we decided to explore the influence of explanations for learning interactions with an equal number of rounds. We considered those children that played the full 30 rounds $(n=22)$, neglecting those who ended the interaction early due to dis-engagement $(n=6)$ or the system's decision that the child's skill belief was good enough $(n=12)$. The sub-sample that played 30 rounds consisted of 22 children, eleven in each condition. Before comparing the groups with regard to learning gain and persistence, we checked the remaining data for normal distribution and homogeneity of variance by means of Shapiro Wilk's and Levene's test. Since the tests were not significant, the preconditions for comparing mean values using a t-test were met for the reduced sub-sample. We thus ran independent sample $t$-tests to compare the mean learning gain and persistence between conditions on the sub-sample, while adjusting alpha using Bonferroni correction to reduce the chance of a Type-I error.

Results showed that children who completed 30 rounds with explanations showed a significantly higher learning gain $(M=3.73, S D=1.27)$ than those who received no explanation $(M=$ $2.18, S D=1.66 ; t_{\text {one-tailed }}(20)=-2.45, p=0.024, d_{\text {Cohen }}=-1.04 ;$ Figures $7(\mathrm{~b})$ and 8$)$. The observed power $^{6}$ for the one-tailed independent samples $t$-test was $63,36 \%$ considering the sub-sample of $n=22$ children (11 per group) that played 30 rounds, with a large effect size of 1.04 and a Bonferroni-corrected alpha of 0.024 . Therefore, although the observed power for the test was moderate, the significant difference $(p<0.05)$ indicates that the sample size was big enough to detect a large effect. Hypothesis H2 was thus at least partly supported. However, we note that this finding is from a follow-up analysis with a reduced sub-sample and that future studies should replicate the finding with higher power to decrease the risk of a Type-II error. With regard to the learning persistence, there was no significant difference between conditions for the sub-sample (see Figure 8).

When reconsidering the remaining participants who quit early due to the system's decision (i.e., a high belief that the child learned enough, $n=12$ ), we observed a high learning gain from pre- to post-test in both groups: with explanations $(n=7, M=4.00, S D=1.73)$ and without $(n=5, M=$ $3.80, S D=2.78)$. After several weeks, in the retention test, this large learning gain diminished a bit

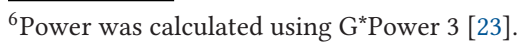


Table 3. Categorization of Target Words According to Children's Perceived Learning

\begin{tabular}{l|c|c|c}
\hline $\begin{array}{l}\text { Categorization of target } \\
\text { words }\end{array}$ & $\begin{array}{c}\text { With Explanation } \\
(\mathbf{n}=\mathbf{1 5})\end{array}$ & $\begin{array}{c}\text { Without Explanation } \\
(\mathbf{n = 1 5 )}\end{array}$ & $\begin{array}{c}\text { Total Cate- } \\
\text { gorizations }\end{array}$ \\
\hline (1) red ("no knowledge") & 21 & 29 & 50 \\
\hline (2) orange ("uncertain") & 15 & 26 & 41 \\
\hline (3) yellow ("rather sure") & 30 & 23 & 53 \\
\hline (4) green ("good knowledge") & 69 & 57 & 126 \\
\hline
\end{tabular}

Frequencies to which children sorted the target words into the given categories. Note that due to a break in the protocol the sorting task was only finished by 30 out of 40 children.

in the explanation group $(M=-0.14, S D=2.55)$ and remained on average the same in the group without explanations $(M=0.00, S D=1.87)$.

Regarding those that quit early due to dis-engagement, we observed that those who learned with explanations did not remember on average more words in the immediate post-test $(n=4$, learning gain: $M=-0.50, S D=1.29)$ but showed an improved learning gain after a couple of weeks $(M=$ $1.25, S D=0.96)$. Instead, those who learned without explanations learned two new words from pre- to post-test $(n=2, M=2.00, S D=0.00)$, while this gain decreased in the retention test $(M=$ $-1.00, S D=0.00)$.

Those children that displayed signs of dis-engagement but continued the interaction until round 30 after re-engagement by the robot $(n=6)$, in contrast, showed a better overall learning gain with explanations $(M=4.00, S D=1.00)$ and without explanations $(M=2.33, S D=2.52)$ than those who quit due to dis-engagement. Regarding persistence, their learning gain slightly increased without explanations $(M=0.67, S D=1.53)$, but diminished over time when explanations were provided $(M=-1.00, S D=2.65)$. Due to the small sub-sample sizes and uneven distributions between the conditions, we refrained from significance testing for the sub groups that finished early.

In summary, the hypothesis that children's learning gain benefits from explanations of the robot's belief and decisions (H2) was supported only for those children, who did not quit early due to dis-engagement or high knowledge, but played the full 30 rounds. In this group, a significantly bigger learning gain was visible when explanations were provided as compared to the control group, lending partial support to our hypothesis (H2).

\subsection{Perceived Learning}

Children's perceived learning was assessed by asking them to categorize taught target words according to no, uncertain, rather sure, or good perceived knowledge (Table 3). Overall, children seemed quite confident and categorized most target words as "good." However, still 50 were categorized under "no knowledge" in total.

To test whether explanations had an impact on the child's estimation of their own knowledge, we first calculated agreement between the children's estimation (low knowledge $=$ categorized as $1 /$ red or 2/orange; high knowledge $=$ categorized as 3/yellow or 4/green) and their word-specific results in the post-test (right/wrong answer). Agreement was given if the child estimated high knowledge and answered correctly in the post-test, or if they estimated the knowledge as low and gave a false answer.

Calculation of an overall agreement between children's estimates and the post-test results reveal on average nearly equal agreements of $64.60 \%(S D=15.39 \%)$ for the with-explanation and $64.67 \%$ $(S D=20.71 \%)$ in the without-explanation condition. We also ran the same analysis only for the sub-sample that did not quit early and played 30 rounds in total. There, children in the explanation condition showed a higher certainty in estimating their perceived learning (64.33\% agreement; $S D=17.49 \%)$ than children in the control group (55.75\% agreement; $S D=20.76 \%$ ). Shapiro Wilk's 
test on normal distribution as well as Levene's test for homogeneity of variances (for the whole sample, and the sub sample) yielded no significance, thus we compared the percentage of agreement between the conditions by means of independent sample t-tests, but the difference was not significant in both cases. H3 was thus not confirmed.

\subsection{Re-engagement After Dis-engagement}

To analyze whether a social robot could effectively get children back into a learning interaction from which they dis-engage (H4a), we analyzed children's motivation to continue interacting after having shown dis-engagement and being addressed by the robot with re-engaging behaviors. In total, twelve children out of 40 exhibited 25 cues of dis-engagement. Half of the dis-engaged children $(n=6)$ were successfully re-engaged by the robot to continue the interaction until the very end (30 rounds). In one case, the first attempt to re-engage the child was unsuccessful and the child quit immediately during or after the robot's attempt. In five other cases, dis-engagement re-occurred a second, third, and fourth time despite of several re-engagement attempts, which also resulted in termination of the interaction. In sum, half of the children who got dis-engaged could be motivated by the robot to continue the learning interaction. H4a is thus at least partially supported. An analysis of whether the success of re-engaging attempts is affected by additional explanation for the triggered action (H4b), reveals that 13 out of $17(76.47 \%)$ attempts were successful in the explanation condition, with six out of eight (75\%) in the control group. That is, there was no significantly higher success of re-engagement actions when accompanied by explanations.

In summary, $30 \%$ of the children received re-engaging actions from the robot, which in turn had an average success rate of $76 \%$. In result, most of the twelve children who showed signs of dis-engagement continued to work in the learning interaction, with six of them sticking to it for the maximum number of rounds. This seems to be independent of the explanation condition.

\section{DISCUSSION}

The overall goal of the present work is to investigate how social robots can be used to scaffold second-language learning of young children aged 4-7 years. In particular, we studied the effects of three scaffolding strategies: adapting the learning task to the child's knowledge state, explaining these decisions, and using re-engagement actions if dis-engagement occurs. We devised a childfriendly learning scenario with a game presented on a tablet computer, augmented with a social robot that guides the child through the interaction as a peer-like tutor. The basis for successful scaffolding is a knowledge-tracing approach that allows for tailoring the interaction to the child's current learning state [71]. Here, we studied if we can provide an additional scaffolding for learning by adding a transparency component, similar to an open learner model [11], that enables the robot to give reasons for the learning tasks based on its beliefs on how the child is doing. In addition, we wanted to know if the robot's ability to produce socially supportive behaviors (e.g., nodding and blinking as positive feedback) can be utilized to increase the children's engagement and, even more challenging, to restore engagement when cues of behavioral dis-engagement were visible.

The results of our study with $n=40$ kindergarten children reveal that adaptive, robot-supported language tutoring is overall successful: all children strongly increased their knowledge from preto post-test. Retention-tests further demonstrated that the knowledge gain persisted for several weeks. Regarding the effects of explanations, the results are faceted in interesting ways: overall, the learning gain for the whole sample did not differ significantly between experimental conditions. A follow up analysis, however, reveals that explanations do have a strong effect on children that completed all 30 rounds: these learners achieved a significantly higher learning gain from pre- to post-test when the robot used explanations, compared to the control group. Since the finding is a result of a follow up analysis, it should be regarded with caution. Due to the reduced sample 
size of 11 per group in the sub-sample, the power of the test was only moderate, but we expect the effect to be even stronger with a larger sample. Moreover, our findings that the means for the whole sample $(n=40)$ were in the hypothesized direction, and the result for the sub-sample of $n=22$ remained significant after Bonferroni correction, lend support to our hypothesis. The large effect size for the test further supports the existence of the difference caused by explanations.

Crucially, the effect does not affect all children equally. While our results demonstrate a positive effect for "slower learners" who finished all 30 rounds and took their time to get familiar with the new words, no such difference emerged for those children who completed the interaction earlier ( $<30$ rounds), because the system decided that their skill was good enough $(>75 \%)$. Since this group reached a similar learning increase as the "slower learners" with explanations, they can be regarded as "fast learners" with a fast knowledge increase after few rounds. These "fast learners" performed equally well with or without additional explanation by the robot. Likewise, the group of "low engagers" who quit early due to dis-engagement, even performed worse if the interaction was lengthened by explanations. Note that we could not provide strict inferential statistics regarding the latter groups due to small sub-sample sizes. Future work should thus explore the influence of explanations for children with high performance and dis-engagement with larger sample sizes.

One possible explanation for the positive impact on slow learners' achievements is that the verbal explanations mitigated their uncertainty about the robot's beliefs, their own performance, or the next tasks to be taken-hence, reducing cognitive load. In other words, in case of nonoptimal performance or increased uncertainty, the robot's explanations can provide a scaffold [27] by reducing felt uncertainty and thus foster learning. However, although explanations affected the learning gain of slow learners in the expected direction, we did not observe any impact on perceived learning. Fast learners, in contrast, who may have a higher task- or learning-proficiency, probably did not work in their "Zone of Proximal Development" [84], and thus do not need additional scaffolding provided by the robot [27]. They already started with higher prior knowledge, performed better and got more positive feedback. Their correspondingly higher affective and cognitive learning may have resulted in a better perceived learning and learning progress. This increase in perceived learning might have further reduced uncertainty, leading to an even smaller effect of the explanations, and resulting in comparable learning gains with and without explanation. For low engagers, however, additional explanations seem to further distract children and, thus, negatively impact learning. Due to their low engagement, these children probably did not pay enough attention to the robot and the interaction as a whole and, thus, were not able to benefit from the provided explanations. Furthermore, the robot's longer speech acts might have distracted or bored them, which in turn might have resulted in even worse learning results compared to those interactions without explanations.

These additional analyses on sub-samples provide fruitful insights, which, however, need further validation in future studies that will be designed to compare the learning gain with larger group sizes under equal interaction times. With regard to the fast learners and low engagers, we even refrained from calculating inferential statistics due to the small sample sizes and uneven distributions between the groups. The interplay of transparency and dis-engagement should hence be explicitly addressed in future studies.

With respect to engagement, our data revealed a positive effect on the overall engagement and motivation of the kindergarten children, as evidenced by longer interaction times and fewer dis-engagement compared to previous study within the same setting [20]: the use of socially supportive behaviors and re-engaging actions to repair dis-engagement, in addition to the robot's normal behavior including iconic gestures for the introduction of new words or adapting the tutoring interaction, led to an overall more engaging and thus more concentrated and longer learning interaction. Interestingly, the robot's re-engagement actions, such as waving or asking the child 
to stand up and stretch the arms, showed a success rate of $76 \%$, with $50 \%$ of the children who started to become dis-engaged continuing the interaction up to the maximum of 30 rounds after a re-engagement attempt by the robot. Furthermore, we observed that those who continued the interaction after re-engagement actions demonstrated a good learning gain, indicating that their dis-engagement was (temporarily) cured. Note that the robot directly asked the children after each re-engagement action whether they wanted to quit or continue the session, and children had to agree to continue. This indicates that the presence of a social robot can be leveraged to maintain the children's commitment to the interaction and the task.

Another reason for the children's willingness to continue could be that the particular reengagement actions mitigated their negative affective state (e.g., tiredness or boredom). Allowing for bodily action such as standing up or stretching arms could have led to more concentration, as necessary for cognitive learning, and to engagement as necessary for affective learning. In addition, the robot's reactions to dis-engagement unveil the robot's active role in keeping track of the learner. In that way, the robot demonstrates a capacity not only to keep track of the child's knowledge state but also to be attentive to their affective state. Knowing that the robot can notice distraction or inattentiveness might have additionally fostered children's effort to concentrate on the task. Yet, making this ability even more salient by use of verbal explanation did not increase this effect. However, future studies should validate the effectiveness of these re-engagement strategies by comparison with a control group that, although showing dis-engagement behavior, does not receive any such strategies.

In summary, we can conclude that social robots can indeed be used to scaffold second-language learning of young children. Specifically, adapting the course of the interaction and the robot's behavior, both to the cognitive state and the engagement state of the child, and explaining these adaptations provide effective support to children who struggle with the learning task or start to get dis-engaged. It is this zone where scaffolding is particularly important and where learning occurs. This also shows that the full potential of a robot tutoring system capable of such strategies can only be exploited when the system is able to respond to the individual differences of children. Future systems should feature a more detailed learner model to profile the children and their needs and to allow for fine-grained adaptation. Such a model should incorporate the child's knowledge state, engagement level, general attributes such as learning speed, as well as indicators of the child's responsiveness to specific strategies (e.g., entries about the success of a given strategy such as gestures or explanations). While such features have been demanded before in the realm of intelligent tutoring systems [26], we have provided here insights into how social robots that can add a new element to the learning environment, can support the different dimensions of learning and what they need to adapt to in this. Still, some effects were just observable for small groups of children and future studies with greater power are needed to further investigate these finding. Moreover, our findings address learning in short-term interactions (such as, e.g., fast mapping processes). They should be complemented with studies on robot-assisted language learning in the proposed setup over a longer period and several tutoring sessions. Additionally, the threshold used to end the training stage early due to a good learning performance needs further fine tuning. An important feature of our system is that this threshold is completely flexible and can be freely adjusted, e.g., dependent on learner characteristics or task demands. It would thus be desirable to vary this threshold in further studies to see which value makes sure that the learner succeeds in hard tasks for every skill, which is not guaranteed with the current study design. Finally, ancillary research will need to test other scaffolding strategies (e.g., reducing supportive behavior) and investigate their influence on learning as well as side effects of strategy combinations. For example, previous findings suggest that the combination of adapting the course of the interaction with iconic gesturing by the robot resulted in a higher learning outcome compared to using each strategy alone [20]. 
Combining an extended learner profile with systematic knowledge about the effects of different supportive strategies (and combinations thereof) should thus be a major goal for the future development of educational social robots that need to respond to the individual needs of each learner in an optimal manner.

\section{APPENDIX}

\section{A PROCEDURE OF TUTORING INTERACTION}

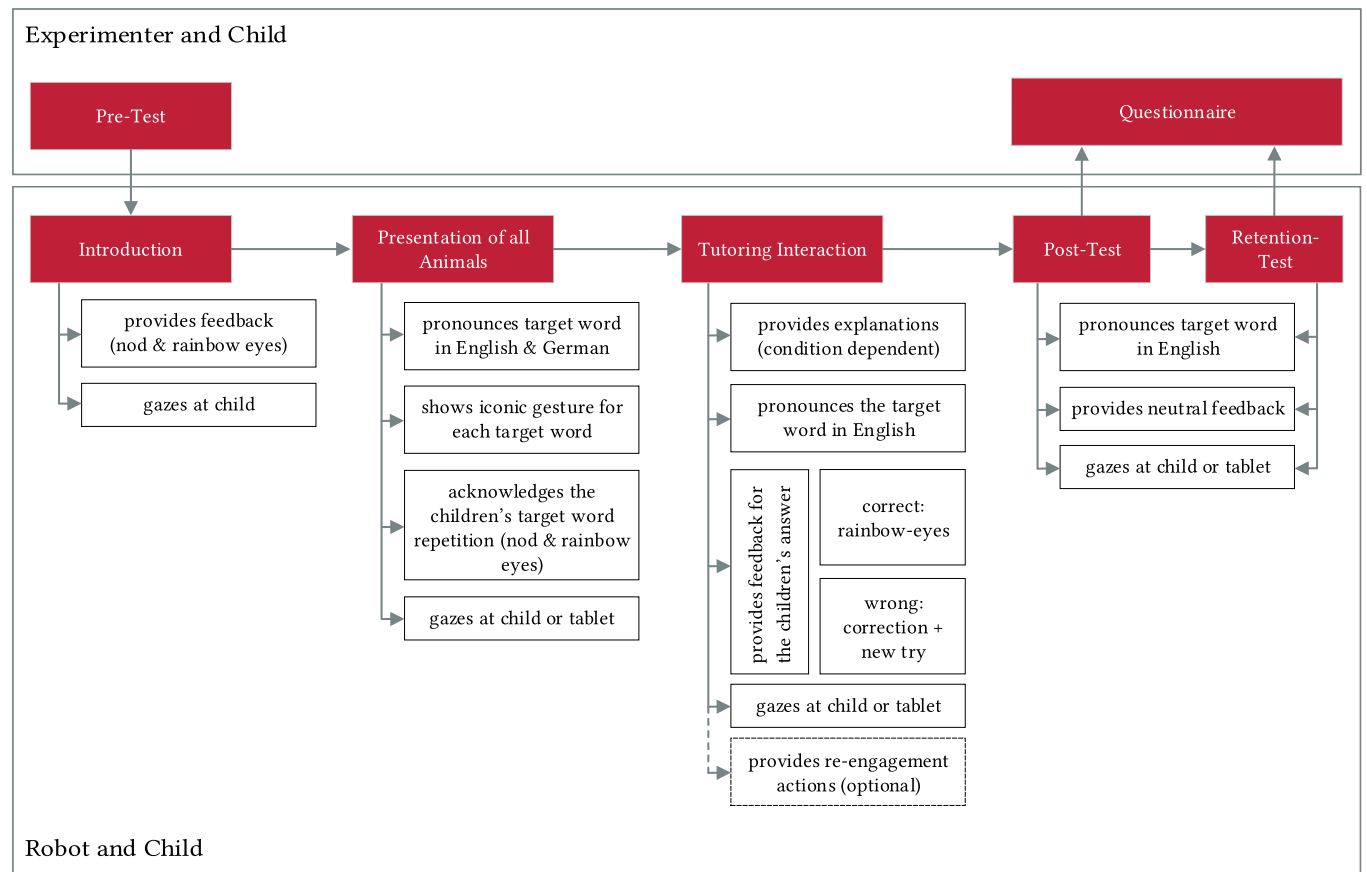

Fig. 9. Overview of the experimental procedure. Red boxes represent the different stages during data collection, and white boxes represent the behaviors shown by the robot in the respective stage of the interaction.

\section{REFERENCES}

[1] Maryam Alavi, George M. Marakas, and Youngjin Yoo. 2002. A comparative study of distributed learning environments on learning outcomes. Info. Syst. Res. 13, 4 (2002), 404-415. DOI : https://doi.org/10.1287/isre.13.4.404.72

[2] Ivon Arroyo, David G. Cooper, Winslow Burleson, Beverly Park Woolf, Kasia Muldner, and Robert Christopherson. 2009. Emotion sensors go to school. In Proceedings of the International Conference on Artificial Intelligence in Education (AIED’09), Vol. 200. 17-24.

[3] Albert Bandura. 1991. Self-regulation of motivation through anticipatory and self-reactive mechanisms. In Proceedings of the Nebraska Symposium on Motivation: Perspectives on Motivation, Vol. 38. 69-164.

[4] Albert Bandura. 1997. Self-efficacy: The Exercise of Control (2nd ed.). Worth Publishers, New York, NY.

[5] Irene M. Barrow, Donald Holbert, and Michael P. Rastatter. 2000. Effect of color on developmental picture-vocabulary naming of 4-, 6-, and 8-year-old children. Speech-Lang. Pathol. 9, 4 (2000), 310-318.

[6] Tony Belpaeme, James Kennedy, Aditi Ramachandran, Brian Scassellati, and Fumihide Tanaka. 2018. Social robots for education: A review. Sci. Robot. 3 (2018), eaat5954.

[7] Tony Belpaeme, Paul Vogt, Rianne van den Berghe, Kirsten Bergmann, Tilbe Göksun, Mirjam de Haas, Junko Kanero, James Kennedy, Aylin C. Küntay, Ora Oudgenoeg-Paz, Fotios Papadopoulos, Thorsten Schodde, Josje Verhagen, Christopher D. Wallbridge, Bram Willemsen, Jan de Wit, Vasfiye Geçkin, Laura Hoffmann, Stefan Kopp, Emiel Krahmer, Ezgi Mamus, Jean-Marc Montanier, Cansu Oranç, and Amit Kumar Pandey. 2018. Guidelines for 
designing social robots as second-language tutors. Soc. Robot. 10, 3 (June 2018), 325-341. DOI : https://doi.org/10.1007/ s12369-018-0467-6

[8] Benjamin S. Bloom, Max D. Engelhart, Edward J. Furst, Walker H. Hill, and David R. Krathwohl. 1956. Taxonomy of Educational Objectives, Handbook I: Cognitive Domain (2nd ed.). Addison-Wesley Longman Ltd, Boston, MA.

[9] Jerome Bruner. 1978. The role of dialogue in language acquisition. Child's Concept. Lang. 2, 3 (1978), 241-256.

[10] Susan Bull, Tim J. Jackson, and Michael J. Lancaster. 2010. Students' interest in their misconceptions in first-year electrical circuits and mathematics courses. Electr. Eng. Edu. 47, 3 (2010), 307-318. DOI : https://doi.org/10.7227/IJEEE. 47.3.6arXiv:https://doi.org/10.7227/IJEEE.47.3.6

[11] Susan Bull and Judy Kay. 2010. Open learner models. In Advances in Intelligent Tutoring Systems, Roger Nkambou, Jacqueline Bourdeau, and Riichiro Mizoguchi (Eds.). Springer, Berlin, 301-322. DOI: https://doi.org/10. 1007/978-3-642-14363-2_15

[12] Maya Cakmak and Manuel Lopes. 2012. Algorithmic and human teaching of sequential decision tasks. In Proceedings of the 26th AAAI Conference on Artificial Intelligence. 1536-1542. Retrieved from http://dl.acm.org/citation.cfm?id= 2900929.2900946.

[13] Benjamin Clement, Didier Roy, Pierre-Yves Oudeyer, and Manuel Lopes. 2015. Multi-armed bandits for intelligent tutoring systems. Edu. Data Min. 7, 2 (2015), 20-48. Retrieved from http://arxiv.org/abs/1310.3174.

[14] Albert T. Corbett and John R. Anderson. 1994. Knowledge tracing: Modeling the acquisition of procedural knowledge. User Model. User-Adapt. Interact. 4, 4 (Dec. 1994), 253-278. DOI : https://doi.org/10.1007/BF01099821

[15] Scotty Craig, Arthur Graesser, Jeremiah Sullins, and Barry Gholson. 2004. Affect and learning: An exploratory look into the role of affect in learning with AutoTutor. Edu. Media 29, 3 (2004), 241-250. DOI: https://doi.org/10.1080/ 1358165042000283101

[16] Nils Dahlbäck, Arne Jönsson, and Lars Ahrenberg. 1993. Wizard of oz studies: Why and how. In Proceedings of the 1st International Conference on Intelligent User Interfaces. 193-200. DOI : https://doi.org/10.1145/169891.169968

[17] Sarah Daily. 2013. Young Children's Self-Regulated Learning and Supportive Teacher-Child Interactions: An Exploratory Study. Ph.D. Dissertation. George Mason University, VA.

[18] Janet E. Davidson and Robert Jeffrey Sternberg. 2003. The Psychology of Problem Solving. Cambridge University Press, Cambridge, UK.

[19] Jacqueline A. de Nooijer, Tamara van Gog, Fred Paas, and Rolf A. Zwaan. 2013. Effects of imitating gestures during encoding or during retrieval of novel verbs on children's test performance. Acta Psychologica 144, 1 (2013), 173-179. DOI : https://doi.org/10.1016/j.actpsy.2013.05.013

[20] Jan de Wit, Thorsten Schodde, Bram Willemsen, Kirsten Bergmann, Mirjam de Haas, Stefan Kopp, Emiel Krahmer, and Paul Vogt. 2018. The effect of a robot's gestures and adaptive tutoring on children's acquisition of second-language vocabularies. In Proceedings of the 13th Annual ACM/IEEE International Conference on Human-Robot Interaction. 50-58. DOI : https://doi.org/10.1145/3171221.3171277

[21] Laurence Devillers and Laurence Vidrascu. 2006. Real-life emotions detection with lexical and paralinguistic cues on human-human call center dialogs. In Proceedings of the 9th International Conference on Spoken Language Processing. 801-804.

[22] Sidney D’Mello and Art Graesser. 2009. Automatic detection of learner's affect from gross body language. Appl. Artific. Intell. 23, 2 (2009), 123-150. DOI : https://doi.org/10.1080/08839510802631745

[23] Franz Faul, Edgar Erdfelder, Albert-Georg Lang, and Axel Buchner. 2007. G*Power 3: A flexible statistical power analysis program for the social, behavioral, and biomedical sciences. Behav. Res. Methods 39, 2 (1 May 2007), 175-191. DOI : https://doi.org/10.3758/BF03193146

[24] Marina Fridin. 2014. Kindergarten social assistive robot: First meeting and ethical issues. Comput. Hum. Behav. 30 (Jan. 2014), 262-272. DOI : https://doi.org/10.1016/j.chb.2013.09.005

[25] Marina Fridin. 2014. Storytelling by a kindergarten social assistive robot: A tool for constructive learning in preschool education. Comput. Edu. 70 (Jan. 2014), 53-64. DOI : https://doi.org/10.1016/j.compedu.2013.07.043

[26] Lorella Giannandrea and Marilena Sansoni. 2013. A literature review on intelligent tutoring systems and on student profiling. Learn. Teach.ing with Media Technol. 287 (2013), 287-294.

[27] Pauline Gibbons. 2002. Scaffolding Language, Scaffolding Learning. Heinemann, Berkeley, CA.

[28] Goren Gordon and Cynthia Breazeal. 2015. Bayesian active learning-based robot tutor for children's word-reading skills. In Proceedings of the 29th AAAI Conference on Artificial Intelligence. 1343-1349. Retrieved from http://dl.acm. org/citation.cfm?id=2887007.2887193.

[29] Goren Gordon, Samuel Spaulding, Jacqueline Kory Westlund, Jin J. Lee, Luke Plummer, Marayna Martinez, Madhurima Das, and Cynthia Breazeal. 2016. Affective personalization of a social robot tutor for children's secondlanguage skills. In Proceedings of the 30th AAAI Conference on Artificial Intelligence. 3951-3957.

[30] Joan Gorham. 1988. The relationship between verbal teacher immediacy behaviors and student learning. Commun. Edu. 37, 1 (1988), 40-53. DOI : https://doi.org/10.1080/03634528809378702 
[31] Jacqueline Gottlieb, Pierre-Yves Oudeyer, Manuel Lopes, and Adrien Baranes. 2013. Information-seeking, curiosity, and attention: computational and neural mechanisms. Trends Cogn. Sci. 17, 11 (2013), 585-593. DOI : https://doi.org/ 10.1016/j.tics.2013.09.001

[32] Barbara A. Greene and Raymond B. Miller. 1996. Influences on achievement: Goals, perceived ability, and cognitive engagement. Contemp. Edu. Psychol. 21, 2 (1996), 181-192. DOI : https://doi.org/10.1006/ceps.1996.0015

[33] Jeonghye Han, Miheon Jo, Sungju Park, and Sungho Kim. 2005. The educational use of home robots for children. In Proceedings of the IEEE International Workshop on Robot and Human Interactive Communication (RO-MAN'05). 378383. DOI : https://doi.org/10.1109/ROMAN.2005.1513808

[34] Stephen R. Hooper and Warren Umansky. 2009. Young Children with Special Needs. Pearson Merrill Prentice Hall, Upper Saddle River, NJ.

[35] Eun-ja Hyun, So-yeon Kim, Siekyung Jang, and Sungju Park. 2008. Comparative study of effects of language instruction program using intelligence robot and multimedia on linguistic ability of young children. In Proceedings of the 17th IEEE International Symposium on Robot and Human Interactive Communication. 187-192.

[36] Mary Helen Immordino-Yang and Antonio Damasio. 2007. We feel, therefore we learn: The relevance of affective and social neuroscience to education. Mind, Brain, Edu. 1, 1 (2007), 3-10. DOI : https://doi.org/10.1111/j.1751-228X.2007. 00004.x

[37] Karin Jampert, Kerstin Leuckenfeld, Anne Zehnbauer, and Petra Best. 2006. Sprachliche Förderung der Kita: Wie viel Sprache Steckt in Musik, Bewegung, Naturwissenschaften und Medien? verlag das netz, Kiliansroda, Germany.

[38] Aidan Jones, Susan Bull, and Ginevra Castellano. 2018. "I know that now, I'm going to learn this next" promoting self-regulated learning with a robotic tutor. Int. F. Soc. Robot. 10, 4 (Sep. 2018), 439-454. DOI : https://doi.org/10.1007/ s12369-017-0430-y

[39] Aidan Jones and Ginevra Castellano. 2018. Adaptive robotic tutors that support self-regulated learning: A longerterm investigation with primary school children. Soc. Robot. 10, 3 (June 2018), 357-370. DOI : https://doi.org/10.1007/ s12369-017-0458-z

[40] Takayuki Kanda, Takayuki Hirano, Daniel Eaton, and Hiroshi Ishiguro. 2004. Interactive robots as social partners and peer tutors for children: A field trial. Hum.-Comput. Interact. 19, 1 (June 2004), 61-84. D0I : https://doi.org/10.1207/ s15327051hci1901\&2_4

[41] Tanja Käser, Severin Klingler, Alexander Gerhard Schwing, and Markus Gross. 2014. Beyond knowledge tracing: Modeling skill topologies with Bayesian networks. In Proceedings of the 12th International Conference on Intelligent Tutoring Systems. 188-198.

[42] James Kennedy, Paul Baxter, and Tony Belpaeme. 2015. The robot who tried too hard: Social behaviour of a robot tutor can negatively affect child learning. In Proceedings of the 10th Annual ACM/IEEE International Conference on Human-Robot Interaction. 67-74. DOI : https://doi.org/10.1145/2696454.2696457

[43] James Kennedy, Paul Baxter, and Tony Belpaeme. 2017. Nonverbal immediacy as a characterisation of social behaviour for human-robot interaction. Soc. Robot. 9, 1 (Jan. 2017), 109-128. DOI : https://doi.org/10.1007/s12369-016-0378-3

[44] James Kennedy, Paul Baxter, Emmanuel Senft, and Tony Belpaeme. 2016. Social robot tutoring for child secondlanguage learning. In Proceedings of the 11th ACM/IEEE International Conference on Human-Robot Interaction. 231-238. DOI : https://doi.org/10.1109/HRI.2016.7451757

[45] Jacqueline Kory and Cynthia Breazeal. 2014. Storytelling with robots: Learning companions for preschool children's language development. In Proceedings of the 23rd IEEE International Symposium on Robot and Human Interactive Communication. 643-648. DOI : https://doi.org/10.1109/ROMAN.2014.6926325

[46] Jacqueline Kory Westlund, Goren Gordon, Samuel Spaulding, Jeongseok Lee, Luke Plummer, Marayna Martinez, Madhurima Das, and Cynthia Breazeal. 2015. Learning a second language with a socially assistive robot. In Proceedings of the 1st International Conference on Social Robots in Therapy and Education.

[47] Hatice Kose-Bagci, Ester Ferrari, Kerstin Dautenhahn, Dag Sverre Syrdal, and Chrystopher L. Nehaniv. 2009. Effects of embodiment and gestures on social interaction in drumming games with a humanoid robot. Adv. Robot. 23, 14 (2009), 1951-1996. DOI : https://doi.org/10.1163/016918609X12518783330360

[48] David R. Krathwohl. 2002. A revision of bloom's taxonomy: An overview. Theory Into Pract. 41, 4 (2002), $212-218$. DOI : https://doi.org/10.1207/s15430421tip4104_2

[49] David R. Krathwohl, Benjamin S. Bloom, and Bertram B. Masia. 1964. Taxonomy of Educational Objectives, the classification of educational goals-Handbook II: Affective Domain. McKay, New York.

[50] Dieter Krowatschek, Caroline Reid, and Gita Krowatschek. 2011. Marburger Konzentrationstraining (MKT) für Schulkinder. Verlag modernes lernen Borgmann, Dortmund. Retrieved from http://widgets.ebscohost.com/prod/ customerspecific/s7170641/vpn.php?url=https://search.ebscohost.com/login.aspx?direct=true \&db=cat04376a\&AN= $\mathrm{kdh} .307154 \&$ lang $=$ de\&site $=$ eds-live.

[51] George D. Kuh. 2003. What we're learning about student engagement from NSSE: Benchmarks for effective educational practices. Change: Mag. Higher Learn. 35, 2 (2003), 24-32. DOI : https://doi.org/10.1080/00091380309604090 
[52] Blair Lehman, Sidney D’Mello, and Natalie Person. 2010. The intricate dance between cognition and emotion during expert tutoring. In Proceedings of the 10th International Conference on Intelligent Tutoring Systems. 1-10.

[53] Iolanda Leite, Marissa McCoy, Daniel Ullman, Nicole Salomons, and Brian Scassellati. 2015. Comparing models of disengagement in individual and group interactions. In Proceedings of the 10th Annual ACM/IEEE International Conference on Human-Robot Interaction. 99-105. DOI : https://doi.org/10.1145/2696454.2696466

[54] Sebastian Leitner. 1972. So Lernt Man Lernen: Der Weg Zum Erfolg [Learning to learn: The road to success]. Herder, Freiburg, Germany.

[55] Miriam Leuchter. 2014. Anschlussfähige Bildungskonzepte für pädagogische Fachkräfte und Grundschullehrpersonen. Carl Link, Munchen, 29-42.

[56] Daniel Leyzberg, Samuel Spaulding, Mariya Toneva, and Brian Scassellati. 2012. The physical presence of a robot tutor increases cognitive learning gains. In Proceedings of the 34th Annual Meeting of the Cognitive Science Society. 1882-1887.

[57] Yanjin Long and Vincent Aleven. 2013. Supporting students' self-regulated learning with an open learner model in a linear equation tutor. In Proceedings of the 2 nd International Conference on Artificial Intelligence in Education. 219-228.

[58] Joseph B. Lyons. 2013. Being transparent about transparency: A model for human-robot interaction. In Proceedings of the AAAI Spring Symposium Series. 48-53.

[59] Joseph B. Lyons, Garrett G. Sadler, Kolina Koltai, Henri Battiste, Nhut T. Ho, Lauren C. Hoffmann, David Smith, Walter Johnson, and Robert Shively. 2017. Shaping trust through transparent design: Theoretical and experimental guidelines. In Advances in Human Factors in Robots and Unmanned Systems, Pamela Savage-Knepshield and Jessie Chen (Eds.). Vol. 499. Springer International Publishing, Basel, Switzerland, 127-136.

[60] Bethany McDaniel, Sidney D’Mello, Brandon King, Patrick Chipman, Kristy Tapp, and Art Graesser. 2007. Facial features for affective state detection in learning environments. In Proceedings of the 29th Annual Meeting of the Cognitive Science Society. 467-472.

[61] Joseph E. Mercado, Michael A. Rupp, Jessie Y. C. Chen, Michael J. Barnes, Daniel Barber, and Katelyn Procci. 2016. Intelligent agent transparency in humanâagent teaming for multi-uxv management. Hum. Fact. 58, 3 (2016), 401-415. DOI : https://doi.org/10.1177/0018720815621206

[62] Raymond B. Miller, Barbara A. Greene, Gregory P. Montalvo, Bhuvaneswari Ravindran, and Joe D. Nichols. 1996. Engagement in academic work: The role of learning goals, future consequences, pleasing others, and perceived ability. Contemp. Edu. Psychol. 21, 4 (1996), 388-422. DOI: https://doi.org/10.1006/ceps.1996.0028

[63] Antonija Mitrovic. 2010. Modeling domains and students with constraint-based modeling. In Advances in Intelligent Tutoring Systems. Springer, 63-80.

[64] Javier R. Movellan, Micah Eckhardt, Marjo Virnes, and Angelica Rodriguez. 2009. Sociable robot improves toddler vocabulary skills. In Proceedings of the 4th ACM/IEEE International Conference on Human-Robot Interaction. 307-308. DOI : https://doi.org/10.1145/1514095.1514189

[65] Neil Naiman, Maria Fröhlich, H. H. Stern, and Angie Todesco. 1996. The Good Language Learner/. Modern languages in practice; 4, Vol. 4. Multilingual Matters, Bristol, PA. Retrieved from http://search.ebscohost.com/login.aspx?direct= true \&scope $=$ site $\& \mathrm{db}=$ nlebk $\& \mathrm{AN}=16738$.

[66] Frank Pajares and M. David Miller. 1994. Role of self-efficacy and self-concept beliefs in mathematical problem solving: A path analysis. Edu. Psychol. 86, 2 (1994), 193-203. DOI: https://doi.org/10.1037/0022-0663.86.2.193

[67] Anna N. Rafferty, Emma Brunskill, Thomas L. Griffiths, and Patrick Shafto. 2011. Faster teaching by POMDP planning. In Proceedings of 15th International Conference on Artificial Intelligence in Education. 280-287.

[68] Aditi Ramachandran, Chien-Ming Huang, Edward Gartland, and Brian Scassellati. 2018. Thinking aloud with a tutoring robot to enhance learning. In Proceedings of the 13th ACM/IEEE International Conference on Human-Robot Interaction. 59-68. DOI : https://doi.org/10.1145/3171221.3171250

[69] Martin Saerbeck, Tom Schut, Christoph Bartneck, and Maddy D. Janse. 2010. Expressive robots in education: Varying the degree of social supportive behavior of a robotic tutor. In Proceedings of the SIGCHI Conference on Human Factors in Computing Systems. 1613-1622. DOI: https://doi.org/10.1145/1753326.1753567

[70] Katherine F. Schetz and Andrew J. Stremmel. 1994. Teacher-assisted computer implementation: A vygotskian perspective. Early Edu. Dev. 5, 1 (1994), 18-26. DOI : https://doi.org/10.1207/s15566935eed0501_2

[71] Thorsten Schodde, Kirsten Bergmann, and Stefan Kopp. 2017. Adaptive robot language tutoring based on Bayesian knowledge tracing and predictive decision-making. In Proceedings of the 12th Annual ACM/IEEE International Conference on Human-Robot Interaction. 128-136. DOI : https://doi.org/10.1145/2909824.3020222

[72] Thorsten Schodde, Laura Hoffmann, and Stefan Kopp. 2017. How to manage affective state in child-robot tutoring interactions? In Proceedings of the 2nd International Conference on Companion Technology. 1-6. DOI : https://doi.org/ 10.1109/COMPANION.2017.8287073

[73] Dale H. Schunk. 1987. Peer models and children's behavioral change. Rev. Edu. Res. 57, 2 (1987), 149-174. DOI : https:// doi.org/10.3102/00346543057002149 
[74] Dale H. Schunk and Barry J. Zimmerman. 1997. Developing self-efficacious readers and writers: The role of social and self-regulatory processes. Reading Engage.: Motivat. Read. Integr. Instruct. 34 (1997), 50.

[75] Dale H. Schunk and Barry J. Zimmerman. 2007. Influencing children's self-efficacy and self-regulation of reading and writing through modeling. Read. Writ. Quart. 23, 1 (2007), 7-25. DOI : https://doi.org/10.1080/10573560600837578

[76] Norbert Schwarz. 2000. Emotion, cognition, and decision making. Cogn. Emot. 14, 4 (2000), 433-440. DOI : https://doi. org/10.1080/026999300402745

[77] Birgit Spinath, Frank M. Spinath, Nicole Harlaar, and Robert Plomin. 2006. Predicting school achievement from general cognitive ability, self-perceived ability, and intrinsic value. Intelligence 34, 4 (2006), 363-374. DOI: https://doi.org/10.1016/j.intell.2005.11.004

[78] Fumihide Tanaka and Shizuko Matsuzoe. 2012. Children teach a care-receiving robot to promote their learning: Field experiments in a classroom for vocabulary learning. Hum.-Robot Interact. 1, 1 (July 2012), 78-95. DOI : https://doi.org/ 10.5898/JHRI.1.1.Tanaka

[79] Olivier Villon and Christine Lisetti. 2006. A user-modeling approach to build user's psycho-physiological maps of emotions using bio-sensors. In Proceedings of the 15th IEEE International Symposium on Robot and Human Interactive Communication. 269-276.

[80] Paul Vogt, Mirjam De Haas, Chiara De Jong, Peta Baxter, and Emiel Krahmer. 2017. Child-robot interactions for second-language tutoring to preschool children. Front. Hum. Neurosci. 11, (Mar. 2017), 1-7. DOI : https://doi.org/10. 3389/fnhum.2017.00073

[81] Paul Vogt, Rianne van den Berghe, Mirjam de Haas, Laura Hoffmann, Junko Kanero, Ezgi Mamus, Jean-Marc Montanier, Cansu Oranç, Ora Oudgenoeg-Paz, Daniel H. Garcia, Fotios Papadopoulos, Thorsten Schodde, Josje Verhagen, Chris D. Wallbridge, Bram Willemsen, Jan de Wit, Tony Belpaeme, Tilbe Göksun, Stefan Kopp, Emiel Krahmer, Aylin C. Küntay, Paul Leseman, and Amit K. Pandey. 2019. Second-language tutoring using social robots. a large-scale study. In Proceedings of the 14th Annual ACM/IEEE International Conference on Human-Robot Interaction. 497-505. DOI : https://doi.org/10.1109/HRI.2019.8673077

[82] Anna-Lisa Vollmer, Robin Read, Dries Trippas, and Tony Belpaeme. 2018. Children conform, adults resist: A robot group induced peer pressure on normative social conformity. Sci. Robot. 3 (2018), eaat7111.

[83] Lev S. Vygotsky. 1962. Thought and Language. MIT Press, Cambridge, MA.

[84] Lev S. Vygotsky. 1978. Mind in Society: The Development of Higher Psychological Processes. Harvard University Press, Cambridge, MA.

[85] Johannes Wagner, Jonghwa Kim, and Elisabeth Andre. 2005. From physiological signals to emotions: Implementing and comparing selected methods for feature extraction and classification. In Proceedings of the IEEE International Conference on Multimedia and Expo. 940-943. DOI : https://doi.org/10.1109/ICME.2005.1521579

[86] David Wood, Jerome S. Bruner, and Gail Ross. 1976. The role of tutoring in problem solving. 7. Child Psychol. Psychiatry 17, 2 (1976), 89-100.

[87] David Wood, Jerome S. Bruner, and Gail Ross. 1976. The role of tutoring in problem solving. F. Child Psychol. Psychiatry 17, 2 (1976), 89-100. DOI : https://doi.org/10.1111/j.1469-7610.1976.tb00381.xarXiv:https://onlinelibrary.wiley. com/doi/pdf/10.1111/j.1469-7610.1976.tb00381.x

[88] Barry J. Zimmerman. 2008. Investigating self-regulation and motivation: Historical background, methodological developments, and future prospects. Amer. Edu. Res. F. 45, 1 (2008), 166-183. D0I:https://doi.org/10.3102/ 0002831207312909

Received September 2018; revised September 2019; accepted September 2019 